\title{
Onboard Beam Generation for Multibeam Satellite Systems
}

\author{
Vahid Joroughi, Miguel Ángel Vázquez, Ana I. Pérez-Neira, Senior Member, IEEE, and Bertrand Devillers
}

\begin{abstract}
This paper aims at designing an onboard beam generation process for a hybrid onboard on-ground multibeam satellite architecture. The proposed method offers a good tradeoff between total throughput and feeder link bandwidth requirements compared with pure on-ground systems. Full frequency reuse among beams is considered, and the beamforming at the satellite is designed for supporting interference mitigation techniques. In addition, in order to reduce the payload cost and complexity, this onboard beamforming is assumed to be constant and the same for forward and return link transmissions so that the same array-fed reflector can be used for forward and return links, leading to a substantial reduction of the payload mass. To meet all these requirements, a novel robust minimum mean square error optimization is conceived. The benefits of the considered scheme are evaluated with respect to the current approaches both analytically and numerically. Indeed, we show that with the DVB-RCS and DVB-S2 standards, our proposal allows increasing the total throughput within a range between $6 \%$ and $15 \%$ with respect to other onboard processing techniques in the return and forward link, respectively.
\end{abstract}

Index Terms-Multibeam satellite systems, on-board beam processing, linear precoding, DVB-S2, DVB-RCS.

$\mathbf{T}$ HE increasing demand for fixed broadband data services is an opportunity for satellite industries to target new markets apart from the well-known current ones (i.e. broadcast broadband, emergency communications, ...). In order to cope with higher data traffic demands, satellite system designers are looking for advanced satellite communication architectures. In this context, the use of multiple beams has recently received a lot of attention as a key enabler of next generation high throughput satellite systems. These systems rely on employing a large number of beams instead of a single (global) beam

Manuscript received May 11, 2015; revised November 11, 2015, May 11, 2016, and October 7, 2016; accepted March 8, 2017. This work was supported in part by the Spanish Ministry of Economy and Competitiveness (ELISA) under Project TEC2014-59255-C3-1-R and in part by the Catalan Government under Grant 2014SGR1567. This work was presented at the IEEE Globecom 2013. The associate editor coordinating the review of this paper and approving it for publication was L. Liu.

V. Joroughi is with the Universidade de Vigo, Vigo, Spain (e-mail: vahid.joroughi@gts.uvigo.es).

M. Á. Vázquez is with the Centre Tecnològic de les Telecomunicacions de Catalunya, Barcelona, Spain (e-mail: mavazquez@cttc.es).

A. I. Pérez-Neira is with the Centre Tecnològic de les Telecomunicacions de Catalunya, Barcelona, Spain, and also with the Universitat Politècnica de Catalunya, Barcelona, Spain (e-mail: ana.isabel.perez@upc.edu).

B. Devillers was with the Centre Tecnològic de les Telecomunicacions de Catalunya, Barcelona, Spain (e-mail: bertrand.devillers@gmail.com).

Color versions of one or more of the figures in this paper are available online at http://ieeexplore.ieee.org.

Digital Object Identifier 10.1109/TWC.2017.2687924 in the coverage area. This is beneficial since each beam can have a larger antenna gain-to-noise temperature than in the single beam case and the available spectrum can be reused among spatially separated beams. Furthermore, whenever the satellite systems delivers broadband unicast (i.e. a single user per beam is served) or multicast (i.e. multiple user per beam are served) interactive traffic, the multibeam architecture can support different modulations and code rates for each user depending on the user link quality, leading to a high increase of the overall system throughput.

Nowadays, the system designers target the Terabit multibeam satellite system, i.e. a satellite system offering a Terabit per second capacity. In fact, the goal is to increase the overall spectral efficiency while keeping the payload complexity affordable. One of the main challenges of Terabit satellite systems is how to deal with the large spectral demands of the feeder link (i.e. the bidirectional communication link between the satellite and the service provider), whose bandwidth requirements increase exponentially as it aggregates the traffic of all users, while keeping a full frequency reuse allocation. Recently, some techniques have been proposed in order to optimize the feeder link spectrum resources. Indeed, there is a current tendency for moving the feeder from the Ka band to the $\mathrm{Q} / \mathrm{V}$ band, where there are larger available bandwidths [1]. Unfortunately, in these frequencies the fading is extremely large and more advanced transmitting diversity techniques are needed.

Another option is the use of multiple gateways, which might be adequate in order to reduce the feeder link spectral requirements as they can be equipped with very directive antennas and exploit the spatial diversity while sharing all available spectrum [2], [3]. Nevertheless, the deployment of several gateways increases the cost of the system and; moreover, the interference mitigation techniques result in certain performance degradation [4], [5]. This is due to the fact that the processing must be separated in isolated processing units.

In contrast to the aforementioned satellite architectures, this paper focuses on the hybrid on-board on-ground processing scheme. This promising solution keeps certain operations in the payload so that the amount of required signals from the feeder link are severely reduced. In this way, the satellite does act in transparent mode and it analogically processes the signals, leading to a high reduction of the feeder link bandwidth requirements. Specifically, while the full on-ground beamforming requires a feeder link bandwidth of

$$
B_{\text {feeder link on-ground }}=N B_{\text {beam }},
$$

1536-1276 (C) 2017 IEEE. Personal use is permitted, but republication/redistribution requires IEEE permission.

See http://www.ieee.org/publications_standards/publications/rights/index.html for more information. 
where $N$ is the number of feed elements ${ }^{1}$ and $B_{\text {beam }}$ is the the total available bandwidth that the users employ; the hybrid on-board on-ground processing only requires

$$
B_{\text {feeder link hybrid }}=K B_{\text {beam }},
$$

where $K$ is the number of beams. As a result, the feeder link bandwidth is reduced since for this work we will consider multiple-feed-per-beam architecture where $N>K$. Note that, in contrast to single-feed-per-beam architectures $(N=K)$, in multiple-feed-per-beam architectures beamforming scan losses are negligible [6]. In addition, multiplefeed-per-beam architecture allows a single on-board reflector to emit beams since single-feed-per-beam typically requires multiple antenna reflectors for a contiguous coverage. A more detailed description of the beam process and its feeder link requirements is presented in [7] and [8].

Apart from the feeder link challenge, multibeam satellite systems require a large capacity in the access network. As a matter of fact, in the generated radiation pattern on Earth, adjacent beams create high levels of interference and, therefore, a carefully planned power and frequency reuse among beams must be employed to cope with this increased level of interference. Consequently, beams with adjacent footprint currently operate in different frequency bands or polarizations. In this context, an essential parameter is the number of colors $N_{c}$ in the frequency reuse pattern, which we define as the cardinality of the set of disjoint frequency bands and polarizations used on the cluster of beam footprints which define the coverage area $\left(N_{c} \geq 1\right)$. In fact, the lower the number of $N_{c}$, the higher the overall system bandwidth will be and the higher the interference power levels will be generated.

In order to increase the available bandwidth yet maintain a low multiuser interference, a promising technique is to use full frequency reuse pattern $\left(N_{c}=1\right)$ and resort to interference mitigation techniques. In this way, signals can be precoded and detected before being transmitted and received in order to reduce inter-beam interference [9]. As a result, a considerable improvement of the achievable spectral efficiency can be obtained. To this end, more advanced interference mitigation techniques as precoding in the forward link and multiuser detection or filtering in the return link have been considered in past studies of the European Space Agency (ESA) [9], [10].

Since interference mitigation techniques require large computational resources, they must be carried out on ground. Indeed, larger efficiencies are obtained if not only the precoding and detection are done on ground, but also the beam generation process, as more flexible processing units are available. In other words, if the beamforming is kept fixed on the payload, there is a performance loss compared to the spectral efficiencies obtained by on ground beamforming [11], [12]. However, if the satellite does not perform any beam processing, the feeder link needs a large amount of spectral resources in order to transmit all the user signals. In addition, centralizing signal processing mechanisms on-ground requires a phase calibration loop between satellite and ground segment. Consequently, even though certain degradation is expected

\footnotetext{
${ }^{1}$ The input signals of the antenna array feed assembly located in the payload.
}

with respect to the on-ground operation (i.e. beam generation, precoding and detection are done in the terrestrial segment), in the present work we propose to optimize the on-board beam generation process so that the achievable rates do not severely decrease due to the on-board beam generation and the feeder link traffic is kept low.

Concretely, this paper focuses on obtaining an optimal onboard beam generation when linear minimum mean square error (LMMSE) precoding technique in the forward link and LMMSE detection procedure in the return link are used as interference mitigation techniques. This study foresees the presence of a non-channel-adaptive (fixed) on-board beam processing scheme in order to keep payload complexity low. Thus, the problem becomes more difficult in the presence of this fixed process in the payload. In order to deal with this problem, we use a robust optimization framework so that a fixed beam generation can be obtained despite user link channel variation.

Furthermore, the designs for both the forward and return links results the same, which makes it appropriate for the future multibeam satellite systems since it is expected that the same reflector is employed at the return and forward links leading to a substantial cost and mass reduction of the payload. Note that the variability of the channel is due to the change of position of the users in consecutive time instants and atmospheric fading. Numerical simulations show the benefit of our method, which in some scenarios can increase the spectral efficiency over the $6 \%$ and $15 \%$ for return and forward links, respectively, if the DVB-S2 and DVB-RCS modulation and coding parameters (modcods) are used.

To the best of the authors knowledge, this is the first time the problem of on-board beam generation process is treated not only in the forward but also in the return link. In contrast to our preliminar work [13], where only the forward link was examined, in this paper we focus our attention to the joint forward and return link optimization. In addition, a novel and better robust design is presented based on a more complete description of the channel perturbations. This new scheme is conceived considering a first order perturbation approach. Finally, several detailed evaluations are presented that validate our contribution in detail.

To sum up, the paper contributions are:

- We propose an on-board beamforming scheme that results the same for forward and return links leading to a substantial reduction of the satellite mass and cost.

- This on board processing considers that precoding and filtering is used so that larger gains are obtained with respect to the schemes that only consider the coverage area.

- In addition, this on board beamforming can keep the feeder link bandwidth requirements low yet preserving substantial gains over the coverage area with respect to full on ground techniques.

- The proposed technique is robust and invariant to channel variations and a novel perturbation analysis is performed.

- Numerical simulations are performed in a close-to-real scenario considering a real deployment which leads to an adequate validation. 
The rest of the paper is organized as follows: Section II presents the signal model. A brief introduction of the beam generation process and the problem characteristics are described in section III. Section IV presents a novel fixed onboard beam generation process. Section $\mathrm{V}$ presents a novel robust scheme based on a first order perturbation analysis. Section VI contains a summary of the simulation results, and eventually the conclusions are given in section VI.

Notation: Throughout this paper, the following notations are adopted. Boldface upper-case letters denote matrices and boldface lower-case letters refer to column vectors. $(.)^{H}$, $(.)^{T},(.)^{*}$ and $(.)^{+}$denote a Hermitian transpose, transpose, conjugate and diagonal (with positive diagonal elements ) matrix, respectively. $\mathbf{I}_{N}$ builds $N \times N$ identity matrix and $\mathbf{0}_{K \times N}$ refers to an all-zero matrix of size $K \times N$. If $\mathbf{A}$ is a $N \times N$ matrix, $\mathbf{A}_{1: K}$ refers to taking the $K$ first rows of the matrix $\mathbf{A}$. $(\mathbf{X})_{i j}$ represents the $(i$-th, $j$-th) element of matrix $\mathbf{X}$. If $\mathbf{B}$ is a $N \times N$ matrix, $\mathbf{A} \leq \mathbf{B}$ implies $\mathbf{A}-\mathbf{B}$ is semidefinite negative. A matrix $\mathbf{M}$ is definite negative if the real part of $\mathbf{z}^{H} \mathbf{M z} \leq 0$ for any non-zero $\mathbf{z}$. $\mathbf{a} \prec \mathbf{b}$ means vector a majorizes vector b. Finally, E\{.\} and $\|$.$\| refer to the$ expected value operator and the Frobenius norm, respectively. The operator o corresponds to the Hadamart product which is a componentwise product.

\section{Signal MODEL}

Let us consider a multibeam satellite communication system, where a single geosynchronous satellite with multibeam coverage provides fixed broadband services to a large set of users, typically operating in Ka-band, although L and S band could be considered depending on the scenario. To this end, the satellite is equipped with an array fed reflector antenna whose number of feeds is denoted by $N$. The coverage area is divided into $K$ beams, with

$$
K<N \text {, }
$$

and the users are assumed to be uniformly distributed within the beams. By employing a time division multiplexing access (TDMA) scheme, at each time instant the gateway is serving a total of $K$ single antenna users (i.e. exactly one user per beam), and it is transmitting (receiving) information to (from) the same number of the users through the satellite in the forward (return) link. Note that in return link satellite communications generally operate in a multi-frequency TDMA (MF-TDMA) so that different users of the same beam might be allocated to different sub-bands. For the sake of simplicity and without loss of generality, the rest of the paper considers TDMA for the return link. Remarkably, the conceived technique can be accommodated to the multi-band communication by replicating the linear processing at each band due to the frequency flatness of the channel response.

The satellite is assumed to linearly convert a set of $N$ onboard feed signals into the $K$ feeder link signals which are transmitted to the gateway in a frequency multiplexed fashion. Reciprocally, in the forward link, the same linear processing strategy is used to construct the $N$ feed signals from the $K$ feeder link signals.

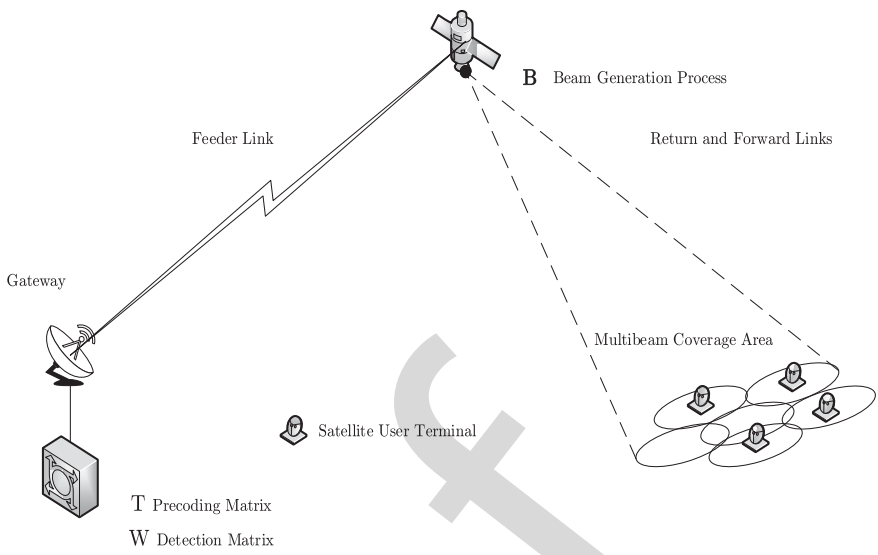

Fig. 1. Multibeam satellite system with on-board beam generation process. The precoding and detection procedures are done on ground. On the contrary, the beam generation process is carried out at the payload and it is assumed to be constant and the same for forward and return links.

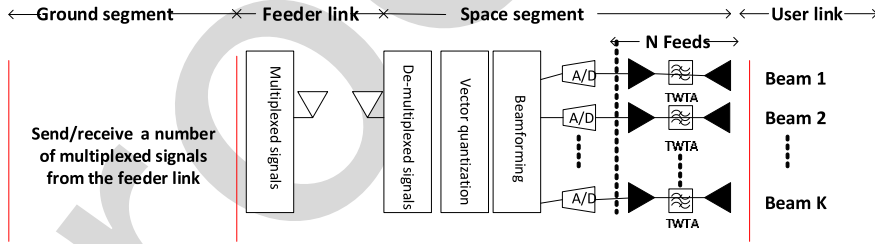

Feeder link: transmits the signals received by gateway to the satellite in a multiplexed fashion Feeds: each feed is shared by multiple beams making TWTAs always operating in multicarrier mode

Fig. 2. Block diagram of an hybrid on-board on-ground multibeam satellite architecture.

Moreover, since a high throughput system is targeted, full frequency reuse among beams is assumed so that all beams can share the same frequency resources. The user link is the communication bottleneck of the whole system. The feeder link is assumed perfectly calibrated and noiseless. Figures 1 and 2 summarize the transmission block diagram.

In the following, the mathematical expressions of the signal model in both the return and forward links are described.

\section{A. Return Link}

As stated above, $K$ denotes the number of users and $N$ is the number of on-board feeds. Then, the corresponding received signal at the gateway can be modelled as

$$
\mathbf{y}_{\mathrm{RL}}=\sqrt{\beta} \mathbf{B H s}+\mathbf{B n},
$$

where $\mathbf{y}_{R L}=\left[y_{R L, 1}, \ldots, y_{R L, K}\right]^{T}$ is a $K \times 1$ vector containing the stack of received signals at the gateway. The $K \times 1$ vector $\mathbf{S}$ is the stack of the transmitted independent signals by all users such that $\mathrm{E}\left\{\mathbf{s s}^{H}\right\}=\mathbf{I}_{K}$. Note that, throughout the paper the subscript $\mathrm{RL}$ is used to refer the return link while $\mathrm{FL}$ will denote the forward link. The constant $\beta$ denotes Equivalent Isotropic Radiated Power (EIRP), which is referred to the user terminal transmit power and we assume to be the same for all the users.

In order to radiate the multibeam pattern, the satellite payload is equipped with a smart antenna system (generally an array fed reflector) coined as on-board beam generation 
process. This system constructs the beam pattern for transmitting and receiving data from the coverage area. Mathematically, the effect of this beamforming appears as the rectangular $K \times N$ fat matrix $\mathbf{B}$.

The $N \times 1$ vector $\mathbf{n}$ accounts for the zero mean Additive White Gaussian Noise (AWGN). We assume unit variance Gaussian noise samples such that

$$
\mathrm{E}\left\{\mathbf{B n n}{ }^{H} \mathbf{B}^{H}\right\}=\mathbf{B B}^{H} .
$$

For radio-frequency design convenience, we will assume that B is orthonormal so that the feed signals are decoupled at the payload $\left(\mathbf{B B}^{H}=\mathbf{I}_{K}\right)$. Matrix $\mathbf{H}$ is the overall $N \times K$ user link channel matrix whose element $\mathrm{h}_{i j}$ presents the aggregate gain of the link between the $i$-th satellite feed and the $j$-th user (in the $j$-th beam). This channel can be decomposed as follows:

$$
\mathbf{H}=\mathbf{G D},
$$

where:

- G is a $N \times K$ matrix that models the feed radiation pattern, the on-board attenuation and path losses. It is responsible for the interference among users. We assume the elements of $\mathbf{G}$ are normalized so that they have unit variance.

- D is assumed to be a $K \times K$ diagonal matrix which takes into account the atmospheric fading in the user link.

Note that $(k, n)$-th entry of the feed radiation pattern matrix $\mathbf{G}$ can be described as follows

$$
(G)_{k, n}=\frac{G_{R} a_{k n}}{4 \pi \frac{d_{k}}{\lambda} \sqrt{K_{B} T_{R} B_{W}}},
$$

with $d_{k}$ the distance between the $k$-th user terminal and the satellite. $\lambda$ is the carrier wavelength, $K_{B}$ is the Boltzmann constant, $B_{W}$ is the carrier bandwidth, $G_{R}^{2}$ the user terminal receive antenna gain, and $T_{R}$ the receiver noise temperature. The term $a_{k n}$ refers to the gain from the $n$-th feed to the $k$-th user. It is important to mention that the $\mathbf{G}$ matrix has been normalized to the receiver noise term. The reader can refer to [12] for a more detailed description of the channel model.

\section{B. Forward Link}

Analogously to the return link, the signal model of the forward link becomes

$$
\mathbf{y}_{\mathrm{FL}}=\gamma \mathbf{H}^{T} \mathbf{B}^{T} \mathbf{x}+\mathbf{w},
$$

where $K \times 1$ vector $\mathbf{y}_{\mathrm{FL}}$ is the stack of received signals at each user terminal, and $\mathbf{x}$ is a $K \times 1$ vector that contains the stack of transmitted symbols. Remarkably, in general wireless communication systems, the channel reciprocity does not hold as uplink and downlink operate in disjoint frequency bands. However, considering our channel modelling, the channel matrix in the forward link differs from the return link in the path loss, feed gain and atmospheric fading. As a result, a scaling factor $\gamma$ can model the different frequency operations.

Similarly as in the return link, $\mathbf{w}$ is a $K \times 1$ vector that represents the independent and identically distributed zero mean Gaussian random noise with unit variance such that

$$
E\left\{\mathbf{w} \mathbf{w}^{H}\right\}=\mathbf{I}_{K} .
$$

Evidently, B does not influence in the forward link noise covariance matrix. We assume the following average available power constraint:

$$
\operatorname{trace}\left(\mathbf{x x}^{H}\right) \leq P_{F L},
$$

where $P_{\mathrm{FL}}$ denotes the total transmit power in the forward link. Note that the transmit power constraint is set without considering the beam generation process $\mathbf{B}$. This is because the power allocation mechanism is located before the array fed reflector system. In addition, it is assumed that the feeds can share the available transmit power. This can be implemented with flexible travelling wave tube amplifiers jointly with multiport power amplifiers as described in [14].

Now, we proceed to jointly optimize matrix $\mathbf{B}$ so that the overall system performance is improved. It is important to remark that $\mathbf{B}$ must be the same for both the optimization of the return and forward links in order to reduce the payload cost. In addition, this matrix needs to be constant in order to keep the payload complexity low and minimize the feeder link spectral resources.

\section{PROBLEM Formulation}

Let us assume that the gateway has perfect Channel State Information (CSI) and uses LMMSE as described in [15] for precoding in the forward link and LMMSE filtering for multiuser detection in the return link. These techniques have been pointed out as efficient methods due to both its interference rejection capabilities and fairness among beams while preserving a low computational complexity [16].

This work resorts to the minimization of the trace of the MSE matrix both at the forward and return links that results from the use of LMMSE precoding and detection. Let us briefly outline the overall mathematical derivation:

1) First, the MSE matrix of the return link is computed assuming LMMSE detection.

2) Second, the MSE matrix of the forward link is computed assuming LMMSE precoding.

3) Third, an upper bound of the MSE minimization in the return link is presented.

4) Finally, a novel robust beam generation process in the return link, which considers the aforementioned upper bound is obtained. For the forward link case, the optimal design yields to the same solution as it is described.

Remarkably, the design of the optimal $\mathbf{B}$ is imposed to be non channel dependent. We show that the optimal $\mathbf{B}$ in the forward and return links results to be the same; thus, fulfilling one of the constraints of the system.

\section{A. Return Link}

As a first step, let us define $\mathbf{W}^{H}$ as the LMMSE filter that detects $K$ received signals at the gateway such that $\hat{\mathbf{s}}=\mathbf{W}^{H} \mathbf{y}_{\mathrm{RL}} ;$ composed by $\hat{\mathrm{s}}_{i}$ which denotes the $i$-th element of the detected signal (for $i$-the user) in the gateway. In this context, the MSE of $i$-th user is achieved as follows

$$
\operatorname{MSE}_{\mathrm{RL}, i}=\mathrm{E}\left\{\left|\mathrm{s}_{i}-\hat{\mathrm{s}}_{i}\right|^{2}\right\},
$$


where $\mathrm{s}_{i}$ represents the $i$-th element of transmit signal vector (for $i$-the user) for a total of $K$ users such that $\mathbf{s}=$ $\left(s_{1}, \ldots, s_{K}\right)^{T}$.

It is well known that the mathematical expression of LMMSE filter becomes

$$
\mathbf{W}^{H}=\left(\mathbf{I}_{K}+\beta \mathbf{H}^{H} \mathbf{B}^{H} \mathbf{B H}\right)^{-1} \mathbf{H}^{H} \mathbf{B}^{H},
$$

and the MSE matrix after the use of this filter is

$$
\left.\mathbf{M S E}_{\mathrm{RL}}=\left(\mathbf{I}_{K}+\beta \mathbf{H}^{H} \mathbf{B}^{H}(\mathbf{B B})^{H}\right)^{-1} \mathbf{B H}\right)^{-1} .
$$

Without loss of generality, we restrict $\mathbf{B}$ to be orthonormal such that $\mathbf{B B}^{H}=\mathbf{I}_{K}$. The sum of MSE in the return link is defined as

$$
\operatorname{SMSE}_{\mathrm{RL}}=\operatorname{trace}\left(\left(\mathbf{I}_{K}+\beta \mathbf{H}^{H} \mathbf{B}^{H} \mathbf{B H}\right)^{-1}\right) .
$$

Now, let us assume for a moment that $\mathbf{B}$ can be channel adaptive (i.e the payload can modify $\mathbf{B}$ depending on the channel variations). Then, the corresponding problem is formulated as

$$
\begin{aligned}
& \min _{\mathbf{B}} \operatorname{trace}\left(\left(\mathbf{I}_{K}+\beta \mathbf{H}^{H} \mathbf{B}^{H} \mathbf{B} \mathbf{H}\right)^{-1}\right) \\
& \text { s.t. } \mathbf{B B}^{H}=\mathbf{I}_{K} .
\end{aligned}
$$

It is important to remark that the authors in [11] showed that the presence of $\mathbf{B}$ increases the $\mathrm{SMSE}_{\mathrm{RL}}$ in the gateway. Mathematically,

$$
\begin{aligned}
& \operatorname{trace}\left(\left(\mathbf{I}_{K}+\beta \mathbf{H}^{H} \mathbf{B}^{H} \mathbf{B} \mathbf{H}\right)^{-1}\right) \\
& \geq \operatorname{trace}\left(\left(\mathbf{I}_{K}+\beta \mathbf{H}^{H} \mathbf{H}\right)^{-1}\right) .
\end{aligned}
$$

Indeed, in [11] it was shown that with the following Singular Value Decomposition (SVD) of the channel $\mathbf{H}=\mathbf{U} \boldsymbol{\Phi} \mathbf{V}^{H}$, an optimal design of $\mathbf{B}$ can be worked out as

$$
\mathbf{B}=\mathbf{U}_{1: K}^{H},
$$

where $\mathbf{U}_{1: K}^{H}$ denotes the $K$ first rows of the matrix $\mathbf{U}^{H}$. In fact, it can be easily seen that this particular solution reaches equality in (16) and; thus, minimizes the $\mathrm{SMSE}_{\mathrm{RL}}$.

In the present work, $\mathbf{B}$ is assumed to be non-channel adaptive, therefore, the design of $\mathbf{B}$ in (17) cannot be considered. Even though the channel appears to be variable at each realization, we aim at finding the best possible non-channel adaptive design of $\mathbf{B}$. In this context, let us decompose the channel as follows

$$
\mathbf{H} \triangleq \overline{\mathbf{H}}+\boldsymbol{\Delta},
$$

where:

- $\overline{\mathbf{H}}$ represents the mean value of the channel.

- $\Delta$ models the difference between the actual value of the channel and its mean. It indicates the variability of the channel in consecutive time instants as already explained in section I.

We assume that the actual channel $\mathbf{H}$ lies in the neighborhood of a nominal channel $\overline{\mathbf{H}}$ that is known to the gateway.
In particular, we consider that $\mathbf{H}$ belongs to the uncertainty region $\mathcal{H} \triangleq\{\mathbf{H}:\|\mathbf{H}-\overline{\mathbf{H}}\| \leq \alpha\}$ which is an sphere centered at $\overline{\mathbf{H}}$ with the radius $\alpha$.

Interestingly, the channel model in (18) resembles the modeling of a MIMO system with imperfect CSI at the transmitter which has been solved as a worst case optimization problem in [17]-[19]. With this perspective for the return link, the worst case robust design is proposed, which leads to a maximin or minimax formulation:

$$
\begin{aligned}
& \min _{\mathbf{B}} \max _{\Delta} \operatorname{trace}\left(\left(\mathbf{I}_{K}+\beta \mathbf{H}^{H} \mathbf{B}^{H} \mathbf{B} \mathbf{H}\right)^{-1}\right) \\
& \text { s.t. } \mathbf{B B}^{H}=\mathbf{I}_{K} .
\end{aligned}
$$

Prior to obtaining the solution of (19), let us focus on the forward link optimization problem, which is similarly derived.

\section{B. Forward Link}

In the forward link, the zero forcing precoding with a regularized inversion is assumed [15]. In this case, the linear precoding is expressed as

$$
\mathbf{x}=\mathbf{T c},
$$

where $\mathbf{T}$ is the $K \times K$ precoding matrix at the gateway and c is the $K \times 1$ transmit symbol vector at all feeds such that $\mathrm{E}\left\{\mathbf{c c}^{H}\right\}=\mathbf{I}_{K}$. In this context, the corresponding precoding matrix $\mathbf{T}$ is expressed as

$$
\mathbf{T}=\gamma \sqrt{\rho} \mathbf{B}^{*} \mathbf{H}^{*}\left(\frac{K}{P_{\mathrm{FL}}} \mathbf{I}_{K}+\gamma^{2} \mathbf{H}^{T} \mathbf{B}^{T} \mathbf{B}^{*} \mathbf{H}^{*}\right)^{-1},
$$

where the value of the constant $\rho$ has to comply with the forward link power constraint as follows

$$
\operatorname{trace}\left(\mathbf{T T}^{H}\right) \leq P_{\mathrm{FL}} .
$$

It is important to remark that in order to properly decode the transmitted symbols, the receivers shall know $\rho$ a prior so that the transmitter shall share this value to all receivers jointly with the transmitted frame. This can be done with during the pilot symbol transmission where precoding is not applied.

This particular kind of precoder is used to find an optimal balance between achieving signal gain and limiting the multiuser interference. Similar to the return link, $\mathrm{MSE}_{\mathrm{FL}, i}$ is defined as

$$
\operatorname{MSE}_{F L, i}=\mathrm{E}\left\{\left|\mathrm{c}_{i}-\hat{\mathrm{c}}_{i}\right|^{2}\right\},
$$

where $\mathrm{MSE}_{\mathrm{FL}, i}$ refers to the MSE received by $i$-th user. Similarly, $\mathbf{c}=\left(c_{1}, \ldots, c_{K}\right)^{T}$ and $\hat{\mathbf{c}}=(\sqrt{\rho})^{-1} \mathbf{y}_{\mathrm{FL}}=\left(\hat{c}_{1}, \ldots, \hat{c}_{K}\right)^{T}$ are the transmitted and received signals for $K$ users, respectively. In this context, $c_{i}$ represents the transmitted signal for $i$-the user and $\hat{\mathrm{c}}_{i}$ denotes the signal received by user $i$-th. The MSE matrix in the forward link can be calculated as follows

$$
\mathbf{M S E}_{\mathrm{FL}}=\mathrm{E}\left\{\left((\sqrt{\rho})^{-1} \mathbf{y}_{\mathrm{FL}}-\mathbf{c}\right)\left((\sqrt{\rho})^{-1} \mathbf{y}_{\mathrm{FL}}-\mathbf{c}\right)^{H}\right\},
$$


which can be rewritten as

$$
\begin{aligned}
\mathbf{M S E}_{\mathrm{FL}}=\frac{K}{P_{\mathrm{FL}}}( & \left(\gamma^{2} \mathbf{H}^{T} \mathbf{B}^{T} \mathbf{B}^{*} \mathbf{B}^{T} \mathbf{B}^{*} \mathbf{H}^{*}+\frac{K}{P_{\mathrm{FL}}} \mathbf{I}_{K}\right) \\
& \left.\times\left(\gamma^{2} \mathbf{H}^{T} \mathbf{B}^{T} \mathbf{B}^{*} \mathbf{H}^{*}+\frac{K}{P_{\mathrm{FL}}} \mathbf{I}_{K}\right)^{-2}\right)
\end{aligned}
$$

As in the return link, we concentrate our efforts to minimize the sum of MSE, this is

$$
\mathrm{SMSE}_{\mathrm{FL}}=\operatorname{trace}\left(\mathbf{M S E}_{\mathrm{FL}}\right),
$$

where, recalling that $\mathbf{B B}^{H}=\mathbf{I}_{K}$ and we consider the following property, $\operatorname{trace}(\mathbf{A})=\operatorname{trace}\left(\mathbf{A}^{T}\right)$ where $\mathbf{A}$ is a square matrix, then we have that

$$
\operatorname{SMSE}_{F L}=\frac{K}{P_{\mathrm{FL}}} \operatorname{trace}\left(\left(\gamma^{2} \mathbf{H}^{H} \mathbf{B}^{H} \mathbf{B H}+\frac{K}{P_{\mathrm{FL}}} \mathbf{I}_{K}\right)^{-1}\right) .
$$

The worst case optimization problem thanks to the channel decomposition in (18) can be formulated as follows

$$
\begin{aligned}
& \min _{\mathbf{B}} \max _{\Delta} \operatorname{trace}\left(\left(\gamma^{2} \mathbf{H}^{H} \mathbf{B}^{H} \mathbf{B H}+\frac{K}{P_{\mathrm{FL}}} \mathbf{I}_{K}\right)^{-1}\right) \\
& \text { s.t. } \mathbf{B B}{ }^{H}=\mathbf{I}_{K} .
\end{aligned}
$$

Note that the return link optimization (19) and the forward link one (28) are the same except for a scalar value. In next section we show that both lead to the same optimal design; thus confirming a natural uplink downlink physical duality.

\section{B Optimization}

This section tackles with the main objective of this paper. An optimally designed $\mathbf{B}$ for problems (19) and (28) is presented. Two main steps are followed. The first step provides a brief description of an upper bound for the SMSE. The second step proposes a design for $\mathbf{B}$ such that it minimizes the proposed SMSE upper-bound obtained in the first step. The design is done for the return link and extended to the forward link.

Prior to presenting the optimal design, we need to introduce the next lemma.

Lemma 1: Assuming an arbitrary square matrix $\mathbf{A}$, the next equation holds

$$
\operatorname{trace}\left(\left(\mathbf{I}_{K}+\mathbf{A A}^{H}\right)^{-1}\right)=\operatorname{trace}\left(\left(\mathbf{I}_{K}+\mathbf{A}^{H} \mathbf{A}\right)^{-1}\right) .
$$

Proof: It is a direct consequence of inversion matrix lemma.

By considering $\mathbf{A}=\sqrt{\beta} \mathbf{B H}$, the $\mathrm{SMSE}_{\mathrm{RL}}$ in problem (19) can be rewritten as

$$
\operatorname{trace}\left(\left(\mathbf{I}_{K}+\beta \mathbf{B Z Z B} \mathbf{B}^{H}\right)^{-1}\right)
$$

where $\mathbf{Z}=\mathbf{H} \mathbf{H}^{H}=\overline{\mathbf{H}} \overline{\mathbf{H}}^{H}+\overline{\mathbf{H}} \boldsymbol{\Delta}^{H}+\boldsymbol{\Delta} \overline{\mathbf{H}}^{H}+\boldsymbol{\Delta} \boldsymbol{\Delta}^{H}$ is a $N \times N$ matrix. We propose an upper bound of $\mathrm{SMSE}_{\mathrm{RL}}$ as follows

Theorem 1: The $S M S E_{R L}$ is upper bounded by

$$
\operatorname{trace}\left(\left(\mathbf{I}_{K}+\beta \mathbf{B Z Z B} \mathbf{B}^{H}\right)^{-1}\right) \leq \operatorname{trace}\left(\left(\mathbf{I}_{K}+\beta \mathbf{B} \mathbf{Z} \mathbf{B}^{H}\right)^{-1}\right)
$$

where

$$
\breve{\mathbf{Z}} \triangleq \overline{\mathbf{U}}\left(\overline{\boldsymbol{\Sigma}}-\epsilon_{H} \mathbf{I}_{N}\right)^{+} \overline{\mathbf{U}}^{H},
$$

so that $\overline{\mathbf{H}} \overline{\mathbf{H}}^{H}=\overline{\mathbf{U}} \overline{\mathbf{\Sigma}} \overline{\mathbf{U}}^{H}$ is the eigen-decomposition of matrix $\overline{\mathbf{H}} \overline{\mathbf{H}}^{H}$. The scalar value $\epsilon_{H}$ is defined as

$$
\epsilon_{H} \triangleq 2 \alpha \delta_{\max }(\overline{\mathbf{H}})
$$

where $\delta_{\max }(\mathbf{C})$ denotes the maximum singular value of $\mathbf{C}$ matrix.

Proof: See [18, Sec.7.3.1].

As a result, a worst-case $S_{\text {MSE }}$ can be obtained in practice by using the lower bound $\breve{\mathbf{Z}}$ in lieu of $\mathbf{Z}$. However, it is important to mention that some values of $\alpha$ lead to unfeasible $\mathrm{MSE}_{\mathrm{RL}}$ solutions, that is, for a large value of $\alpha$ the matrix (32) might become low rank since ()$^{+}$operator delivers 0 whenever the diagonal entry is non positive. Due to that, the value of $\alpha$ has to be checked and, if necessary, decreased so that

$$
\operatorname{rank}(\breve{\mathbf{Z}})=K \text {. }
$$

In order to obtain a robust design, the target is to minimize the proposed upper-bound of $\mathrm{SMSE}_{R L}$ in (31) instead of (19). In this case, the corresponding problem is formulated as

$$
\begin{aligned}
& \min _{\mathbf{B}} \operatorname{trace}\left(\left(\mathbf{I}_{K}+\beta \mathbf{B} \breve{\mathbf{Z}} \mathbf{B}^{H}\right)^{-1}\right) \\
& \text { s.t. } \mathbf{B B} \mathbf{B}^{H}=\mathbf{I}_{K} .
\end{aligned}
$$

The solution to this optimization problem is described in the next theorem.

Theorem 2: The upper bound of SMSE is minimized if $\mathbf{B}$ is selected as the first $K$ rows of the matrix $\overline{\mathbf{U}}^{H}$, that is

$$
\mathbf{B}^{\star}=\overline{\mathbf{U}}_{1: K}^{H},
$$

where $\mathbf{B}^{\star}$ denotes the optimal design of $\mathbf{B}$.

Proof: See Appendix A.

Remark: It is important to mention that the derivation of theorem 2 differs to [20, Th. 1]. The main difference consists of the constraint since in [20] a total power constraint is considered

$$
\operatorname{trace}\left(\mathbf{B B}^{H}\right) \leq P_{\mathrm{FL}},
$$

where as this paper assumes

$$
\mathbf{B B}^{H}=\mathbf{I}_{K},
$$

which involves further mathematical developments as described in Appendix A.

Before starting with the forward link case, let us remark that $\mathbf{B}^{\star}$ only needs statistical channel knowledge in order to be computed. Moreover, its design does not depend on $\alpha$ as long as the resulting rank of $\breve{\mathbf{Z}}$ is equal to $K$. Indeed, the value of $\alpha$ affects only on the resulting SMSE $\mathrm{RL}_{\mathrm{RL}}$. This is due to the optimization of an upper bound of the problem instead of the problem itself. Now, let us proceed with the forward link optimization. 
In the forward link the optimization problem can be formulated as follows

$$
\begin{aligned}
& \min _{\mathbf{B}} \operatorname{trace}\left(\left(\gamma^{2} \mathbf{B} \breve{\mathbf{Z}} \mathbf{B}^{H}+\frac{K}{P_{\mathrm{FL}}} \mathbf{I}_{K}\right)^{-1}\right) \\
& \text { s.t. } \mathbf{B B}^{H}=\mathbf{I}_{K} .
\end{aligned}
$$

In can be observed that the optimal solution of (39) is (36). The sketch of the proof is similar to the one presented previously for the return link and; thus, we only comment it. The idea is to check whether the term $\frac{K}{P_{\mathrm{FL}}}$ does not influence the optimal value of (39) which can be easily observed in appendix A. Consequently, neither the scaling factor due to the channel variations $\gamma$ does not influence the optimization. Remarkably, this derivation is different from the one presented in our preliminary work in [13], because this paper considers the forward and return link optimizations.

Note that the beamforming scheme depends on $\overline{\mathbf{H}}$ so that the system designer needs to be aware of it in advance. This information can be obtained through current deployments or estimations. In addition, the robust beamforming design has the same eigenvectors as the nominal channel matrix $\overline{\mathbf{H}} \overline{\mathbf{H}}^{H}$. In other words, the presented robust design only considers eigenvalue variations due to the different user positions. In the next section, the impact on the eigenvectors is analyzed.

\section{First Order Perturbation Analysis}

Considering the derivations of the previous section, obtaining a robust on-board beamforming matrix leads to computing an accurate upper-bound of $\mathbf{Z}$ considering the different sources of perturbation. This section completes the upper-bound by including an additional impact of the perturbation errors.

A complete perturbation model can be described as

$$
\begin{aligned}
\mathbf{Z}= & \left(\overline{\mathbf{U}}_{s}+\Delta \mathbf{U}_{s}\right)\left(\overline{\boldsymbol{\Sigma}}_{s}+\Delta \boldsymbol{\Sigma}_{s}\right)\left(\overline{\mathbf{U}}_{s}+\Delta \mathbf{U}_{s}\right)^{H} \\
& +\left(\overline{\mathbf{U}}_{n}+\Delta \mathbf{U}_{n}\right)\left(\overline{\boldsymbol{\Sigma}}_{n}+\Delta \boldsymbol{\Sigma}_{n}\right)\left(\overline{\mathbf{U}}_{n}+\Delta \mathbf{U}_{n}\right)^{H},
\end{aligned}
$$

where the $\mathbf{U}$ denotes the matrix containing the eigenvectors and $\boldsymbol{\Sigma}$ is a diagonal matrix which contains the eigenvalues. Sub-index $s$ denotes the non-zero signal space whereas $n$ the signal space that is spanned by the zero valued eigenvalues (i.e. the null space of $\mathbf{Z}$ ). All $\Delta \mathbf{U}_{s}, \Delta \boldsymbol{\Sigma}_{s}, \Delta \mathbf{U}_{n}, \Delta \boldsymbol{\Sigma}_{n}$ are generated by a perturbed version of $\overline{\mathbf{Z}}$ :

$$
\mathbf{Z}=\overline{\mathbf{Z}}+\Delta \mathbf{Z},
$$

where

$$
\overline{\mathbf{Z}}=\overline{\mathbf{H}} \overline{\mathbf{H}}^{H},
$$

and

$$
\Delta \mathbf{Z}=\overline{\mathbf{H}} \Delta^{H}+\Delta \overline{\mathbf{H}}^{H}+\Delta \Delta^{H} .
$$

Under this context, $\overline{\mathbf{U}}$ denotes the eigenvector of the nominal matrix $\overline{\mathbf{Z}}$ whereas $\overline{\boldsymbol{\Sigma}}$ a matrix containing its eigenvalues. The other matrices with the $\Delta$. prefix denote the corresponding perturbation matrices.

The previous section has implicitly considered two assumptions. First, it has been assumed that the channel variations do not modify the dimension of the null space so that $\Delta \boldsymbol{\Sigma}_{n}$ remains as a zero matrix. Second, it has been assumed that $\Delta \mathbf{U}_{s}=0$, which might not be true in certain cases [21]. The aim of this section is to consider the effect of this later perturbation in order to obtain a more realistic version of $\mathbf{Z}$ than the presented in the previous section, $\widehat{\mathbf{Z}}$.

This novel approximation of $\mathbf{Z}, \widehat{\mathbf{Z}}$, considers both perturbations at both eigenvalues and eigenvectors $\left(\Delta \mathbf{U}_{s}\right)$. Mathematically,

$$
\widehat{\mathbf{Z}}=\left(\overline{\mathbf{U}}_{s}-\mathbf{P}\right)\left(\overline{\mathbf{\Sigma}}-\epsilon_{H} \mathbf{I}\right)\left(\overline{\mathbf{U}}_{s}-\mathbf{P}\right)^{H},
$$

where $\mathbf{P}$ is a semidefinite positive matrix that has the same dimensions of $\mathbf{U}_{s}$. Note that it is essential to obtain a matrix $\mathbf{P}$ that collapses the maximum of the eigenvectors perturbation. In the following we propose a solution in order to properly design the on-board beamforming when eigenvector perturbations are present.

Proposal The beamforming matrix that takes into account both the eigenvalues and eigenvector perturbation can be written as

$$
\widehat{\mathbf{B}^{*}}=\widehat{\mathbf{U}}=\overline{\mathbf{U}}_{s}-\left(\epsilon_{H} \overline{\mathbf{U}}_{s} \widehat{\mathbf{R}}+\epsilon_{H} \overline{\mathbf{U}}_{n} \overline{\mathbf{U}}_{n}^{H} \overline{\mathbf{U}}_{s} \overline{\boldsymbol{\Sigma}}_{s}^{-1}\right),
$$

where

$$
\widehat{\mathbf{R}}=\mathbf{D} \circ\left(\mathbf{U}_{s}^{H} \mathbf{U}_{s} \overline{\boldsymbol{\Sigma}}+\overline{\boldsymbol{\Sigma}} \mathbf{U}_{s}^{H} \mathbf{U}_{s}\right),
$$

and the $g, f$-th entry of $\mathbf{D}$ is

$$
\frac{1}{\lambda_{f}-\lambda_{g}}
$$

for $f \neq g$ and $\lambda_{f}$ for $f=1, \ldots, N$ denote the eigenvalues of $\overline{\mathbf{H}} \overline{\mathbf{H}}^{H}$.

Proof: See Appendix B.

Note that for this case, the eigenvectors of the beamforming matrix take a different value from the nominal matrix. In addition, the larger $\alpha$ the more different are the eigenvectors from the nominal channel matrix ones. However, $\alpha$ cannot take any arbitrary value. Indeed, the permissible perturbation value is dictated by the fact that the resulting matrix containing the eigenvectors shall be semidefinite positive. As a result, $\epsilon_{H}$ shall hold

$$
\overline{\mathbf{U}}_{s} \geq\left(\epsilon_{H} \overline{\mathbf{U}}_{s} \widehat{\mathbf{R}}+\epsilon_{H} \overline{\mathbf{U}}_{n} \overline{\mathbf{U}}_{n}^{H} \overline{\mathbf{U}}_{s} \overline{\boldsymbol{\Sigma}}_{s}^{-1}\right) .
$$

It is important to remark that $\widehat{\mathbf{B}^{*}}$ is not a unitary matrix and; thus, it is not an efficient solution of the optimization problem (39) as it does not satisfies the constraints. However, we take the heuristic approach of electing $\widehat{\mathbf{B}}^{*}$ processing, even though the solution is not unitary since it is obtained from a more detailed description of the perturbation errors impact. This solution is validated in the simulation section and it is observed that it presents a slightly better performance than the $B^{*}$ solution.

As we have already seen, the beam generation process both on the forward and return links leads to the same matrix $\mathbf{B}$, which is fixed. Now, it is time to compare the benefits of this design in front of the current beamforming deployments. 


\section{Simulation Results}

In order to show the performance of our proposal, this section presents a numerical evaluation of the conceived technique. Our baseline scenario is an array fed reflector antenna and matrix $\mathbf{B}$ that have been provided by ESA in the framework of a study on next generation multibeam satellite systems. ${ }^{2}$ The number of feeds is assumed to be $N=155$ and $K=100$ beams that are covering the whole Europe area.

Results have been averaged over a total of 1000 user link channel realizations. Note that, only atmospheric fading due to rain effect is considered in the user link channel and further refinements of the channel are neglected. This simple characterization is useful for the intended comparisons and it is a general practice in the evaluation of multibeam satellite systems.

The randomness of the channel is due to the user positions which are assumed to be uniformly distributed within the beams. In addition, we will assume that each user employs all available spectrum and the atmospheric fading is modelled as in [22].

Recall that, full frequency reuse among beams and noiseless feeder link have been considered in this work. In the sequel, we compute different performance metrics. First, the SINR for each user after employing interference mitigation techniques among users is presented. Then, with that SINR value, the throughput is inferred according to DVB-RCS and DVB-S2 standards for the return and forward links, respectively [23], [24]. Furthermore, the simulation results also provide the SINR statistics. In this case, the instantaneous availability indicator for the $k$-th user is given by

$$
A_{k}=g\left(\operatorname{SINR}_{k}\right)
$$

which is equal to 0 if the user link is unavailable (i.e, if the instantaneous SINR is lower than that required by the lowest modcod for the return link, i.e. $\operatorname{SINR}_{k}<1.7 \mathrm{~dB}$, and for the forward link, i.e. $\operatorname{SINR}_{k}<-2.72 \mathrm{~dB}$ ) and is equal to 1 otherwise. We also present the Shannon capacity ${ }^{3}$ obtained from the user SINR,

$$
C_{\text {Shannon }}=\log _{2}(1+\text { SINR }),
$$

and assuming that interference is treated as Gaussian noise. This measurement serves us to see the potential of our work independently of the satellite standard modulations and channel coding both for the forward and return links.

Another performance metric to be considered is the fairness among beams. Note that this is of great interest for satellite operators where near to equal achievable data rates per beam are the target. For this purpose, we present the throughput index of dispersion, defined as

$$
\text { Index of Dispersion }=\frac{\sigma_{\mathrm{Th}}}{\mu_{\mathrm{Th}}},
$$

where $\sigma_{\mathrm{Th}}$ and $\mu_{\mathrm{Th}}$ correspond to the variance and the mean of the user throughputs, respectively. This metric provides an

\footnotetext{
${ }^{2}$ http://satnex4.org/

${ }^{3}$ Of course, we refer to the use of the Shannon formula instead of the channel capacity.
}

indicator of how the data rates are dispersed with respect to the mean. The larger the index of dispersion is, the lower the fairness the system achieves.

For a best practice, as upper bound for the achievable rates we consider only on-ground processing at the gateway (i.e. no on-board processing) as it is described in [11]. From the return link point of view, the received signal (4), which is based on this on-ground scenario, is rewritten as

$$
\mathbf{y}_{\mathrm{RL}}=\mathbf{T}_{\text {on-ground }}^{H}(\mathbf{H s}+\mathbf{n}),
$$

where

$$
\mathbf{T}_{\text {on-ground }}=\mathbf{H}\left(\mathbf{H H}^{H}+\mathbf{I}_{K}\right)^{-1}
$$

denotes the LMMSE detector filter at the gateway. Note that the linear processing is similar to (12) but in this case it has been assumed that no beamforming is done. Considering the forward link, the received signal by the user terminals with this on-ground technique can be represented as

$$
\mathbf{y}_{\mathrm{FL}}=\mathbf{H}^{T} \mathbf{T}_{\text {on-ground }} \mathbf{x}+\mathbf{w} \text {. }
$$

It is important to remark that although large data rates can be obtained if all the processing is carried out on ground, the required feeder link spectral resources severely increase, leading to a possibly inefficient system.

To sum up, in order to test the validity of the derived theoretical results in section IV, we compute the spectral efficiency of the following multibeam satellite system using precoding and detection algorithms for forward and return links respectively:

- B based on a geographical reasoning (reference). ${ }^{4}$

- $\mathbf{B}^{*}$ proposed by this study in (36).

- $\widehat{\mathbf{B}}^{*}$ proposed by this study in (45).

- On ground processing (upper bound).

In the sequel, the results are separated into two different subsections, return and forward link. In this context, the same fixed optimal design of on-board beamforming matrix is computed since this optimal design depends on the right eigen vector of channel average matrix, $\overline{\mathbf{H}}$. This is computed empirically considering the aforementioned 1000 channel user realizations.

\section{A. Return Link}

The return link operates at $30 \mathrm{GHz}$, and is based on DVBRCS standard [23] and we target a Packet Error Rate (PER) of $10^{-7}$. Figure 3 depicts the evolution of the total average throughput (bits/symbol) as a function of the user EIRP $(\beta)$ for different scenarios. Although by means of using the DVB-RCS standard the obtained throughput gain is limited when the Shannon capacity is considered, higher gains are obtained with respect to the reference scenario. In other words, other modcods design would improve the benefits of the proposed technique with respect to the reference scenario. Note that the proposed robust design that consider the eigenvector perturbation improves the system throughput with

\footnotetext{
${ }^{4}$ This beam generation process has attended geographical reasons, so that ESA confirmed all Europe achieves a sufficient signal power strength.
} 


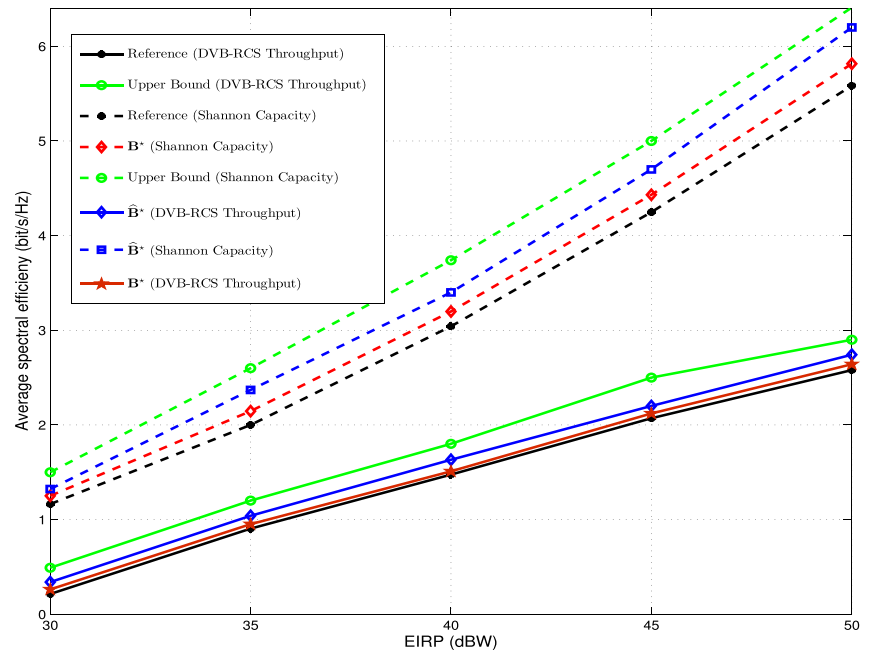

Fig. 3. Return link throughput values over different user EIRP $(\beta)$.

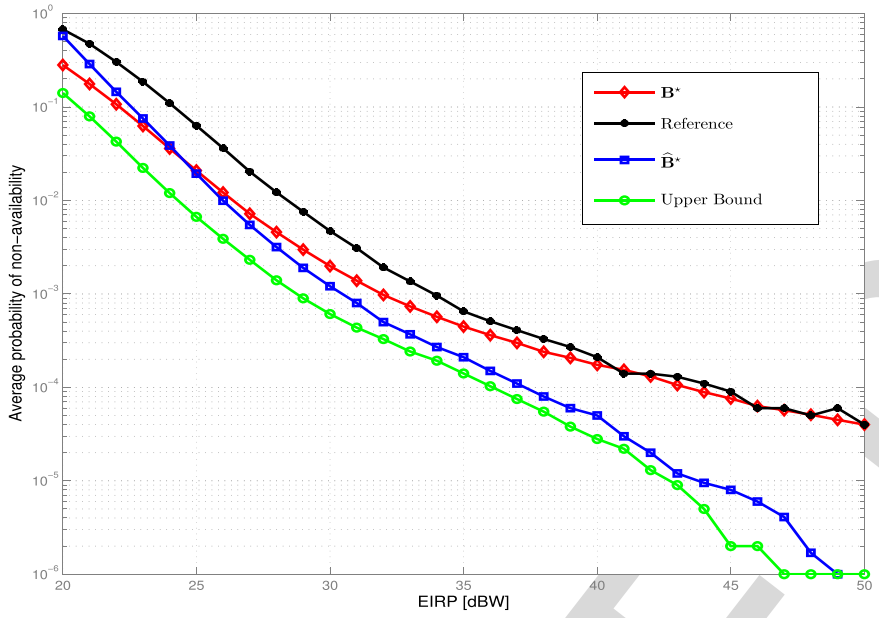

Fig. 4. Return link availability.

respect to the design that only considers eigenvalue variations. Indeed, our proposal is approaching the upper bound of the on ground design.

The corresponding availability probability is also provided in Figure 4. In this case, our proposal also improves the reference scenario, leading to an increase of the system availability. Remarkably, the fairness among beams is also improved as it is depicted in Figure 5. Lower values of dispersion index are obtained with our technique with respect to the reference design.

Finally, we study the impact of the channel variations on the beamforming design. Bearing in mind that $\alpha$ in (33) determines this variation, we compute this value and we present its corresponding average throughput values in Figure 6. The values of $\alpha$ are selected so that the feasibility of $\mathrm{MSE}_{\mathrm{RL}}$ in (32) holds. It implies that

$$
\left(\overline{\bar{\Sigma}}-\epsilon_{H} \mathbf{I}_{N}\right)_{i i} \geq 0 \quad \forall i=1, \ldots, N .
$$

For a large value of $\alpha$ the matrix (55) might become semidefinite negative and; thus, changes the nature of the problem. In order to avoid this, $\alpha$ has to be checked so that the matrix (55) always remains semidefinite positive. It is observed

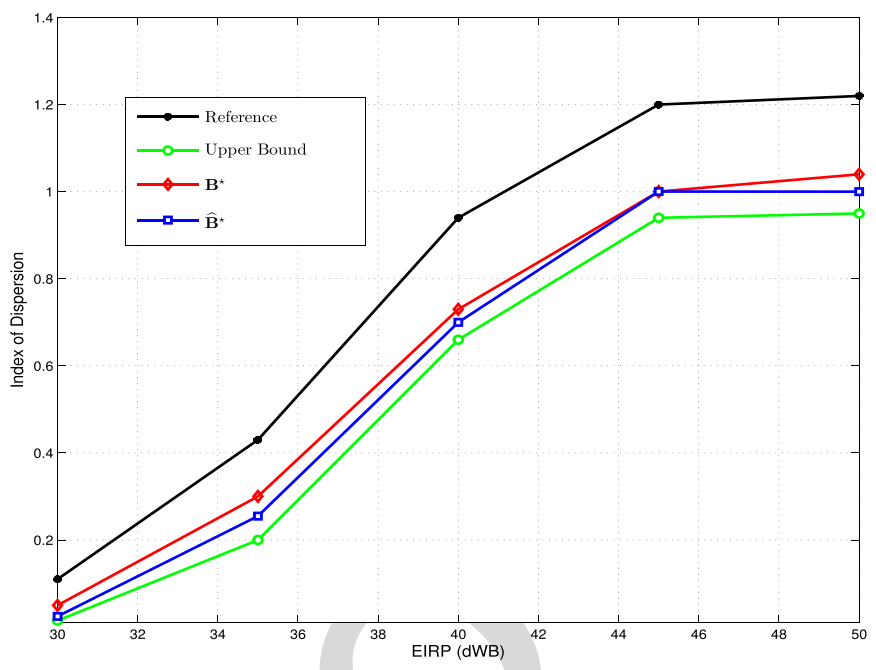

Fig. 5. Return link throughput index of dispersion.

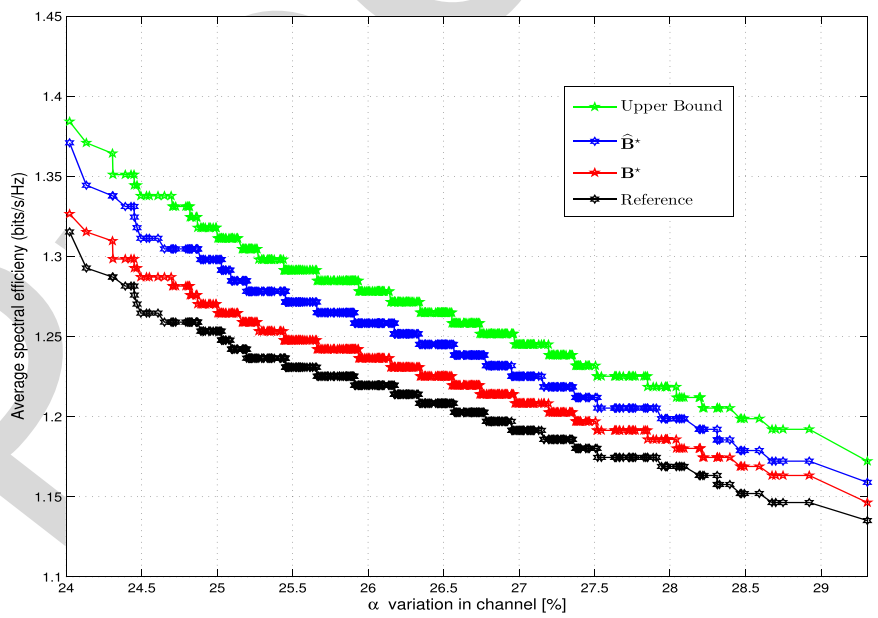

Fig. 6. Return link throughput with respect to channel variations. Note that $\alpha$ determines maximum variation of the channel at each time instant, i.e. $\|\mathbf{\Delta}\| \leq \alpha$.

that the larger $\alpha$ values, the less the throughput is obtained due to the channel mismatch.

\section{B. Forward Link}

The forward link is assumed to operate at $20 \mathrm{GHz}$ and is based on DVB-S2 standard with a PER of $10^{-6}$. Note that the working points were extrapolated from the PER curves reported in the DVB-S2 guidelines document [24]. Based on [24], it is possible to find a relationship between the required received SINR and the spectral efficiency achieved by DVB-S2 standard.

The results are presented for the total bandwidth and as a function of the total available power denoted by $P_{F L}$. Figure 7 depicts the achieved results of spectral efficiency and Figure 8 shows the availability of the users in the forward link. Clearly, the proposed techniques perform better than the benchmark system and again the robust design based on the eigenvector perturbations behaves better than the one that only considers the eigenvalues.

The expected result of throughputs in Figure 7 is justified by the availability in Figure 8. In other words, the system with 


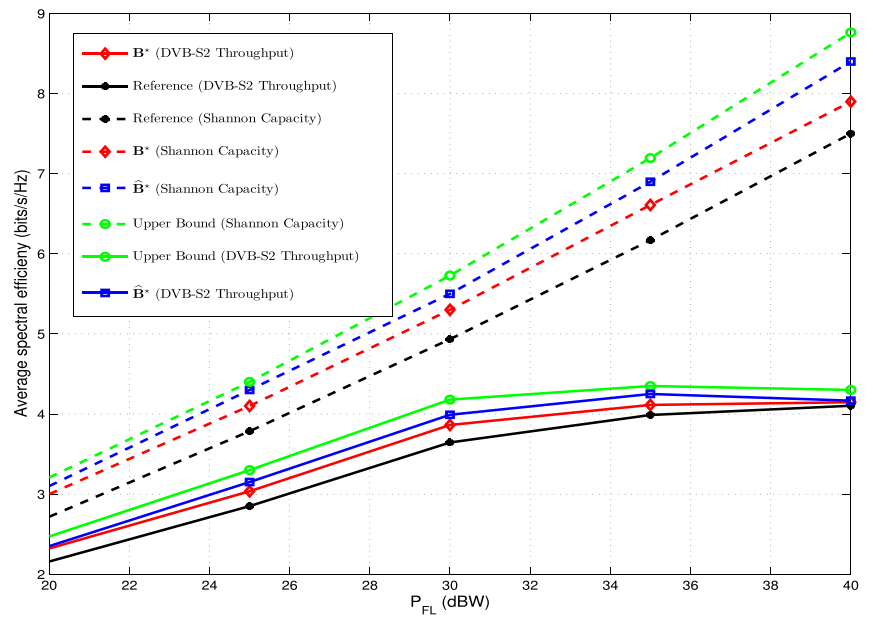

Fig. 7. Forward link throughput values.

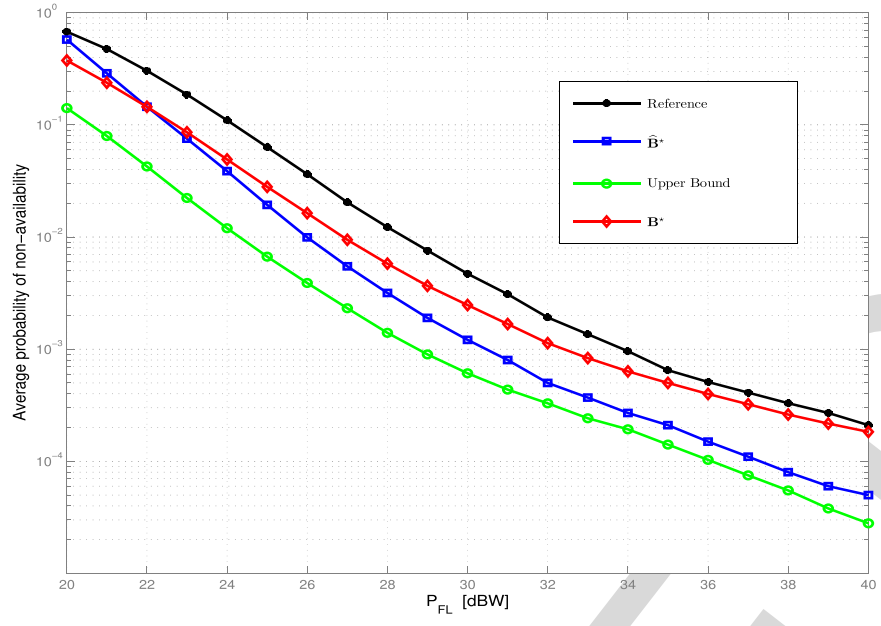

Fig. 8. Forward link availability.

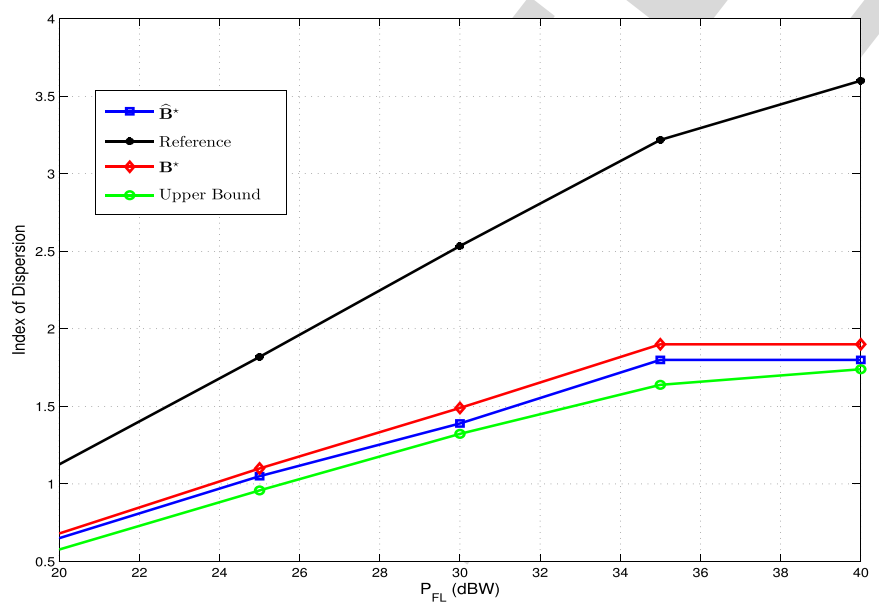

Fig. 9. Forward link throughput index of dispersion. new proposed design of $\widehat{\mathbf{B}}^{*}$ is closer to upper bound scenario than the reference. Moreover, the impact of channel variations can be observed in Figure 10. It is clear that our proposal results in higher throughputs even when the channel variations are high. Remarkably, for the forward link the performance difference is higher than the one obtained in the return link. Note that, similar to the return link, the values of $\alpha$ are selected

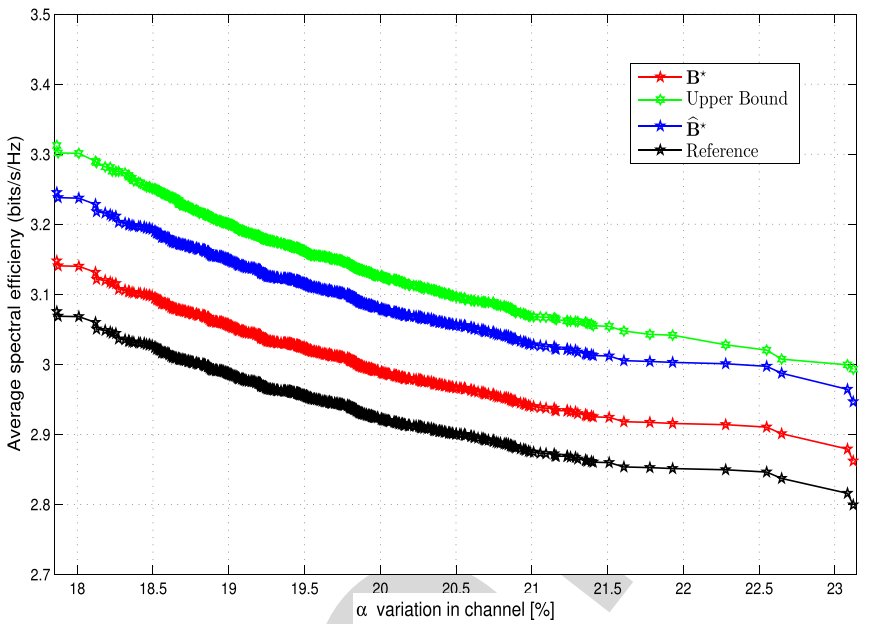

Fig. 10. Forward link throughput with respect to channel variations. Note that $\alpha$ determines maximum variation of the channel at each time instant, i.e. $\|\mathbf{\Delta}\| \leq \alpha$
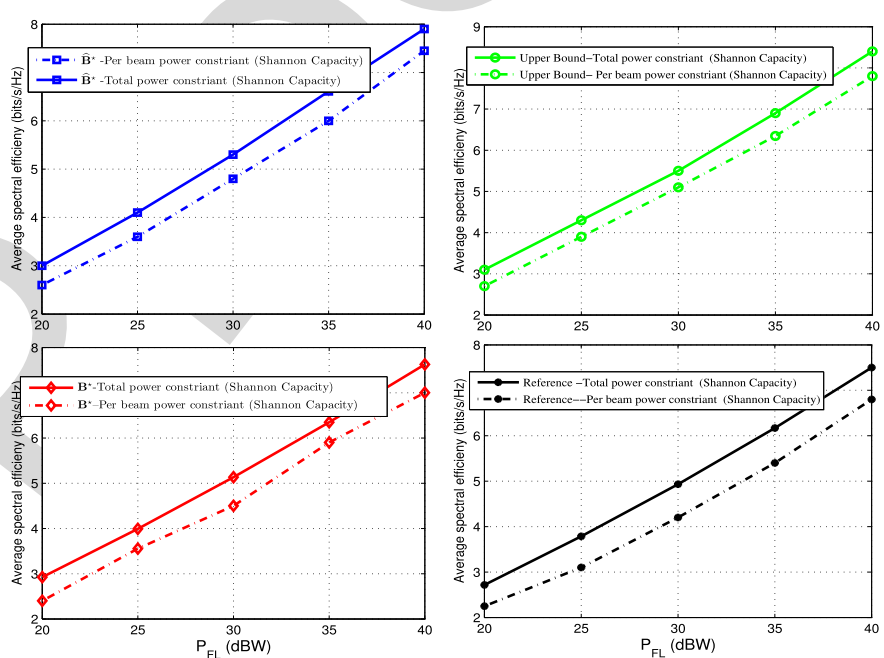

Fig. 11. Forward link throughput with per antenna power constraints.

so that the feasibility of $\mathrm{MSE}_{\mathrm{FL}}$ in (32) holds. Figure 11 describes the effect of per antenna power constraints. It can be observed that the through is severely reduced considering this power allocation. In addition, it has been considered that the available power is equally distributed among feeds. The dispersion index among users is analysed and represented in Figure 9. For this case, the dispersion values are even higher for the reference scenario and our approach leads to higher fairness between beams.

Finally, in order to compare the different satellite architectures we describe in Table I the total capacity values and required feeder link bandwidth for few satellite architectures. The values are obtained for a transmit power of $30 \mathrm{dBW}$ for the forward link with MMSE precoding. Moreover, the hybrid architecture uses the proposed on-board processing based on the eigenvector perturbation scheme.

The parameter $\eta$ is defined as the ratio between the total satellite capacity (i.e. the sum of the rates of all beams) and the feeder link bandwidth, and the colour is defined as the frequency reuse factor within the beam coverage area. In light of the above table, it is evident that on-ground precoding with 
TABLE I

Comparison of Total CAPACITY Versus FeEder LinK BANDWIDTH REQUIREMENTS FOR DIFFERENT MULTIBEAM SATELLITE ARCHITECTURES

\begin{tabular}{|c|c|c|c|}
\hline Architecture & $\begin{array}{c}\text { Total Capacity } \\
(\mathrm{Gbit} / \mathrm{s})\end{array}$ & $\begin{array}{c}B_{\text {feeder link }} \\
(\mathrm{GHz})\end{array}$ & $\begin{array}{c}\eta \\
(\mathrm{bit} / \mathrm{s} / \mathrm{Hz})\end{array}$ \\
\hline On-ground Precoding one colour & 158 & 77.5 & 2.03 \\
\hline On-ground Precoding 2 colours & 107 & 38.5 & 2.77 \\
\hline On-ground Precoding 4 colours & 32 & 19.4 & 1.65 \\
\hline Hybrid one colour with $\widehat{\mathbf{B}}^{*}$ & 137 & 50 & 2.74 \\
\hline
\end{tabular}

2 colours is the most efficient architecture in terms of $\eta$. However, this architecture offers lower system capacity (107 Gbit/s) with respect to the hybrid and on-ground with full frequency reuse (158 and $137 \mathrm{Gbit} / \mathrm{s}$ respectively). As a result, the hybrid architecture is the most adequate solution considering the future traffic demand forecasts. Although the numerical details are not included in this work, the same conclusion can be obtained for the return link part.

It is important to remark that even through the computation of $\eta$ depends on the beampattern, $N$ and $K$ as a general statement we can infer that, given a satellite user available bandwidth (500 MHz for this case), our proposed on-board beamforming technique offers a large throughput yet maintaining a low feeder link bandwidth requirement compared to the other architectures with only on-ground processing. Remarkably, although the 2-colour plus precoding solution offers a larger $\eta$, its system capacity yields to a lower value compared to the hybrid case and; thus, this later solution is the most adequate for next generation multibeam satellite systems.

Finally, the on-board beamforming entails an additional payload processing compared for the pure on-ground approach. This complexity increase could limit its applicability in future systems. Therefore, the system designer could eventually opt to a pure on-ground architecture whose payload complexity is lower compared to the hybrid case. In this context, attending to the system capacity increase, the best option is the one colour plus precoding approach. On the other hand, the 2 colour plus precoding case is the one that offers the largest feeder link efficiency.

\section{CONCLUSION}

This paper proposes a design of non-channel adaptive beam generation process that increases the system throughput compared to the conventional existing techniques in both forward and return link of a multibeam satellite system. The design is based on an upper bound approximation of the worst case SMSE, which results to be the same for both forward and return links, leading to a large reduction of the payload complexity. The robust approximation relies on a first perturbation model which results tighter than current robust designs. Moreover, the simulation results also have shown the potential advantage of the considered design in order to increase the total system throughput. As a consequence, this new approach could become a breakthrough in the design of the next satellite systems, which so far have designed the onboard beamforming only based on geographical information.

\section{APPENDIX A}

The goal is to prove, the proposed optimal design of $\mathbf{B}$ in (36) can minimize the upper-bound of $\mathrm{SMSE}_{\mathrm{RL}}$ in (35). First, by employing the eigenvalue decomposition of $\breve{\mathbf{Z}}$ in (32), problem (35) can be rewritten as

$$
\begin{aligned}
& \min _{\mathbf{M}_{R L}} \operatorname{trace}\left(\left(\mathbf{I}_{K}+\mathbf{M}_{R L} \mathbf{D}_{R L} \mathbf{M}_{R L}^{H}\right)\right)^{-1} \\
& \text { s.t. } \mathbf{M}_{R L} \mathbf{M}_{R L}^{H}=\mathbf{I}_{K},
\end{aligned}
$$

with the following definitions

$$
\mathbf{M}_{R L} \triangleq \mathbf{B} \overline{\mathbf{U}}
$$

and,

$\mathbf{D}_{R L} \triangleq\left(\overline{\boldsymbol{\Sigma}}-\epsilon_{H} \mathbf{I}_{N}\right)^{+}=\left(\begin{array}{cc}\left(\overline{\boldsymbol{\Sigma}}_{1: K}-\epsilon_{H} \mathbf{I}_{K}\right)^{+} & \mathbf{0}_{K \times(N-K)} \\ \mathbf{0}_{(N-K) \times K} & \mathbf{0}_{(N-K) \times(N-K)}\end{array}\right)$,

where $\overline{\boldsymbol{\Sigma}}$ has only $K$ non-zero eigenvalues, as $\overline{\mathbf{H}} \overline{\mathbf{H}}^{H}$ has rank equal to $K$. Actually, the problem (56) can be written as

$$
\begin{aligned}
& \min _{\mathbf{M}_{R L}} \sum_{i=1}^{K} \frac{1}{1+\lambda_{i}\left(\mathbf{M}_{R L} \mathbf{D}_{R L} \mathbf{M}_{R L}^{H}\right)} \\
& \text { s.t. } \mathbf{M}_{R L} \mathbf{M}_{R L}^{H}=\mathbf{I}_{K},
\end{aligned}
$$

where $\lambda_{i}($.$) denotes the i$-th largest eigenvalue of the respective matrix. Obviously, $\mathbf{M D M}^{H}$ is a hermitian matrix whose eigenvalues are always positive. Then, it follows that

$$
g\left(\lambda_{i}\right)=\frac{1}{1+\lambda_{i}\left(\mathbf{M}_{R L} \mathbf{D}_{R L} \mathbf{M}_{R L}^{H}\right)} \quad i=1, \ldots, K ;
$$

is convex function on $\lambda_{i}\left(\mathbf{M}_{R L} \mathbf{D}_{R L} \mathbf{M}_{R L}^{H}\right)$. By using [25, Th. 3.C.1], we have that

$$
\begin{aligned}
\phi(\lambda) & =\sum_{i=1}^{K} \frac{1}{1+\lambda_{i}\left(\mathbf{M}_{R L} \mathbf{D}_{R L} \mathbf{M}_{R L}^{H}\right)} \\
& =\sum_{i=1}^{K} g\left(\lambda_{i}\left(\mathbf{M}_{R L} \mathbf{D}_{R L} \mathbf{M}_{R L}^{H}\right)\right),
\end{aligned}
$$

where $\lambda=\left(\lambda_{1}\left(\mathbf{M}_{R L} \mathbf{D}_{R L} \mathbf{M}_{R L}^{H}\right), \ldots, \lambda_{K}\left(\mathbf{M}_{R L} \mathbf{D}_{R L} \mathbf{M}_{R L}^{H}\right)\right)^{T}$, and $\phi($.$) is a schur-convex function operator. On other hand,$ [25, Th. B.1] proved that

$$
\mathbf{d} \prec \lambda,
$$

where $\mathbf{d}($.$) represents K \times 1$ vector formed by the diagonal elements of the matrix $\mathbf{M}_{R L} \mathbf{D}_{R L} \mathbf{M}_{R L}^{H}$, i.e. $\mathbf{d}=$ $\left(d_{1}\left(\mathbf{M}_{R L} \mathbf{D}_{R L} \mathbf{M}_{R L}^{H}\right), \ldots, d_{K}\left(\mathbf{M}_{R L} \mathbf{D}_{R L} \mathbf{M}_{R L}^{H}\right)\right)^{T}$. Finally, combining of (62) with the schur convexity of $\phi($.$) , we have that$ $\phi(\mathbf{d}) \leq \phi(\lambda)$, i.e.

$$
\sum_{i=1}^{K} \frac{1}{1+d_{i}\left(\mathbf{M}_{R L} \mathbf{D}_{R L} \mathbf{M}_{R L}^{H}\right)} \geq \sum_{i=1}^{K} \frac{1}{1+\lambda_{i}\left(\mathbf{M}_{R L} \mathbf{D}_{R L} \mathbf{M}_{R L}^{H}\right)} .
$$


Moreover, the equality in (63) is reached whenever $\mathbf{M}_{R L} \mathbf{D}_{R L} \mathbf{M}_{R L}^{H}$ is diagonal. To this end, it is clear that $\mathbf{M}$ has to be diagonal such that

$$
\mathbf{M}_{R L}=\left[\begin{array}{ll}
\mathbf{I}_{K} & \mathbf{0}_{K \times(N-K)}
\end{array}\right] .
$$

Note that this differs to the developments in $[20, \mathrm{Sec}$. V] where the diagonal elements are optimized for different objective functions. Our approach is to meet the constraint in (56).

Given (57), it implies that $\mathbf{B}$ has to be made of the $K$ first rows of the matrix $\overline{\mathbf{U}}^{H}$, that is

$$
\mathbf{B}=\overline{\mathbf{U}}_{1: K}^{H},
$$

and concludes the proof.

\section{APPENDIX B}

The starting point of the derivation is the upper bound of $\mathbf{Z}$ obtained when considering only the eigenvalues variation

$$
\overline{\mathbf{U}}_{s}\left(\overline{\boldsymbol{\Sigma}}-\epsilon_{H} \mathbf{I}\right) \overline{\mathbf{U}}_{s}^{H},
$$

where for this case we additionally consider the perturbation on the eigenvectors as

$$
\left(\overline{\mathbf{U}}_{s}+\Delta \mathbf{U}_{s}\right)\left(\overline{\boldsymbol{\Sigma}}-\epsilon_{H} \mathbf{I}\right)\left(\overline{\mathbf{U}}_{s}+\Delta \mathbf{U}_{s}\right)^{H} .
$$

Note that the derivation in order to obtain Theorem 1, remains the same even though the perturbation over the eigenvectors is considered. In [21] it is presented that the perturbation on the eigenvectors take the form of

$$
\Delta \mathbf{U}_{s}=\overline{\mathbf{U}}_{s} \mathbf{R}+\overline{\mathbf{U}}_{n} \overline{\mathbf{U}}_{n}^{H} \Delta \mathbf{Z} \overline{\mathbf{U}}_{s} \overline{\boldsymbol{\Sigma}}_{s}^{-1}
$$

where

$$
\mathbf{R}=\mathbf{D} \circ\left(\mathbf{U}_{s}^{H} \Delta \mathbf{Z} \mathbf{U}_{s} \overline{\boldsymbol{\Sigma}}+\bar{\Sigma} \mathbf{U}_{s}^{H} \Delta \mathbf{Z}^{H} \mathbf{U}_{s}\right),
$$

and the $g, f$-th entry of $\mathbf{D}$ is

$$
\frac{1}{\lambda_{f}-\lambda_{g}},
$$

for $f \neq g$ and $\lambda_{f}$ for $f=1, \ldots, N$ denote the eigenvalues of $\overline{\mathbf{H}} \overline{\mathbf{H}}^{H}$.

The aim of this derivation is to substitute the unknown matrix $\Delta \mathbf{Z}$ by the known matrix $\epsilon_{H} \mathbf{I}$ which models its maximum perturbation value. This derivation entails certain assumptions of the definite positiveness of different matrices that might not hold in general. In any case, the aim of the following work is to motivate and relate the proposed solution with the eigenvector matrix perturbation.

First, we want to show that

$$
\Delta \mathbf{U}_{s} \leq \overline{\mathbf{U}}_{s} \mathbf{R}+\epsilon_{H} \overline{\mathbf{U}}_{n} \overline{\mathbf{U}}_{n}^{H} \overline{\mathbf{U}}_{s} \overline{\boldsymbol{\Sigma}}_{s}^{-1},
$$

in case the following inequality holds

$$
\Delta \mathbf{Z} \leq \epsilon_{H} \mathbf{I} .
$$

In order to show this, we shall assume

$$
\overline{\mathbf{U}}_{n} \overline{\mathbf{U}}_{n}^{H} \Delta \mathbf{Z} \overline{\mathbf{U}}_{s} \overline{\boldsymbol{\Sigma}}_{s}^{-1} \leq \epsilon_{H} \overline{\mathbf{U}}_{n} \overline{\mathbf{U}}_{n}^{H} \overline{\mathbf{U}}_{s} \overline{\boldsymbol{\Sigma}}_{s}^{-1} .
$$

Considering (73) it can be obtained (71).
Additionally, we have to assume

$\mathbf{U}_{s}^{H} \Delta \mathbf{Z} \mathbf{U}_{s} \overline{\boldsymbol{\Sigma}}+\bar{\Sigma} \mathbf{U}_{s}^{H} \Delta \mathbf{Z}^{H} \mathbf{U}_{s} \leq \epsilon_{H} \mathbf{U}_{s}^{H} \mathbf{U}_{s} \overline{\boldsymbol{\Sigma}}+\epsilon_{H} \overline{\boldsymbol{\Sigma}} \mathbf{U}_{s}^{H} \mathbf{U}_{s}$.

The following lemma is required for obtaining the result.

Lemma 2: For any complex matrix $\mathbf{K}$ and two square complex matrices $\mathbf{A} \geq \mathbf{B} \geq \mathbf{0}$, it holds that

$$
\mathbf{K} \circ \mathbf{A} \geq \mathbf{K} \circ \mathbf{B} \text {. }
$$

Proof: This can be derived from in [26, Th. 17].

With this last result it is possible to write the following

$$
\Delta \mathbf{U}_{s} \leq \overline{\mathbf{U}}_{s} \widehat{\mathbf{R}}+\epsilon_{H} \overline{\mathbf{U}}_{n} \overline{\mathbf{U}}_{n}{ }^{H} \overline{\mathbf{U}}_{s} \overline{\boldsymbol{\Sigma}}_{s}^{-1},
$$

where

$$
\widehat{\mathbf{R}}=\mathbf{D} \circ\left(\mathbf{U}_{s}^{H} \Delta \mathbf{Z} \mathbf{U}_{s} \overline{\boldsymbol{\Sigma}}+\bar{\Sigma} \mathbf{U}_{s}^{H} \Delta \mathbf{Z}^{H} \mathbf{U}_{s}\right) .
$$

Note that we have assumed

$$
\epsilon_{H} \mathbf{U}_{s}^{H} \mathbf{U}_{s} \overline{\boldsymbol{\Sigma}}+\epsilon_{H} \overline{\boldsymbol{\Sigma}} \mathbf{U}_{s}^{H} \mathbf{U}_{s} \geq 0 .
$$

In this context, Lemma 2 can be applied.

Considering the inequality in (74) jointly with (76), we can write

$$
\Delta \mathbf{U}_{s} \leq \overline{\mathbf{U}}_{s} \hat{\mathbf{R}}+\epsilon_{H} \overline{\mathbf{U}}_{n} \overline{\mathbf{U}}_{n}{ }^{H} \overline{\mathbf{U}}_{s} \overline{\boldsymbol{\Sigma}}_{s}^{-1},
$$

where

$$
\widehat{\mathbf{R}}=\mathbf{D} \circ\left(\epsilon_{H} \mathbf{U}_{s}^{H} \mathbf{U}_{s} \overline{\boldsymbol{\Sigma}}+\epsilon_{H} \overline{\boldsymbol{\Sigma}} \mathbf{U}_{s}^{H} \mathbf{U}_{s}\right) .
$$

The right side of the inequality in (79) is the one presented in the proposal.

\section{ACKNOWLEDGMENT}

The authors would like to thank the anonymous reviewers whose comments extremely increased the quality of the paper.

\section{REFERENCES}

[1] J.-D. Gayrard, "Terabit Satellite: Myth or reality?" in Proc. 1st Int. Conf. Adv. Satellite Space Commun., (SPACOMM), Jul. 2009, pp. 1-6.

[2] G. Zheng, S. Chatzinotas, and B. E. Ottersten, "Generic optimization of linear precoding in multibeam Satellite systems," IEEE Trans. Wireles Commun., vol. 11, no. 6, pp. 2308-2320, Jun. 2012.

[3] A. Gharanjik, B. Rao, P.-D. Arapoglou, and B. Ottersten, "Gateway switching in Q/V band Satellite feeder links," IEEE Commun. Lett., vol. 17, no. 7, pp. 1384-1387, Jul. 2013.

[4] V. Joroughi, M. A. Vazquez, and A. Perez-Neira, "Multiple gateway precoding with per feed power constraints for multibeam Satellite systems," in Proc. 20th Eur. Wireless Conf., May 2014, pp. 1-7.

[5] G. Zheng, S. Chatzinotas, and B. Ottersten, "Multi-gateway cooperation in multibeam Satellite systems," in Proc. IEEE 23rd Int. Symp. Pers Indoor Mobile Radio Commun. (PIMRC), Sep. 2012, pp. 1360-1364.

[6] M. Schneider, C. Hartwanger, and H. Wolf, "Antennas for multiple spot beam Satellites," CEAS Space J., vol. 2, nos. 1-4, pp. 59-66, 2011. [Online]. Available: http://dx.doi.org/10.1007/s12567-011-0012-z

[7] J. Tronc, P. Angeletti, N. Song, M. Haardt, J. Arendt, and G. Gallinaro, "Overview and comparison of on-ground and on-board beamforming techniques in mobile Satellite service applications," Int. J. Satellite Commun. Netw., no. 4, pp. 291-308, 2013. [Online]. Available: http://dx.doi.org/10.1002/sat.1049

[8] P. Angeletti, N. Alagha, and S. D'Addio, "Space/ground beamforming techniques for Satellite communications," in Proc. IEEE Antennas Propag. Soc. Int. Symp., Jul. 2010, pp. 1-4.

[9] L. Cottatellucci et al., "Interference mitigation techniques for broadband Satellite system," in Proc. ICSSC, San Diego, CA, USA, Jun. 2006, p. 5348. [Online]. Available: http://www.eurecom.fr/publication/1886 
[10] V. Boussemart, M. Berioli, F. Rossetto, and M. Joham, "On the achievable rates for the return-link of multi-beam Satellite systems using successive interference cancellation," in Proc. MILITARY Commun. Conf. MILCOM, Nov. 2011, pp. 217-223.

[11] J. Arnau, B. Devillers, C. Mosquera, and A. Perez-Neira, "Performance study of multiuser interference mitigation schemes for hybrid broadband multibeam Satellite architectures," EURASIP J. Wireless Commun. Netw., vol. 2012, no. 1, p. 132, 2012. [Online]. Available: http://jwcn.eurasipjournals.com/content/2012/1/132

[12] B. Devillers, A. Perez-Neira, and C. Mosquera, "Joint linear precoding and beamforming for the forward link of multi-beam broadband Satellite systems," in Proc. IEEE Global Telecommun. Conf. (GLOBECOM), Dec. 2011, pp. 1-6.

[13] V. Joroughi, B. Devillers, M. Á. Vázquez, and A. I. Pérez-Neira, "Design of an on-board beam generation process for the forw ard link of a multi-beam broadband Satellite system," in Proc. IEEE Global Commun. Conf. (Globecom), Dec. 2013, pp. 2921-2926.

[14] M. Aloisio and P. Angeletti, "Multi-amplifiers architectures for power reconfigurability," in Proc. IEEE Int. Vac. Electron. Conf. (IVEC), May 2007, pp. 1-2.

[15] C. B. Peel, B. M. Hochwald, and A. L. Swindlehurst, "A vectorperturbation technique for near-capacity multiantenna multiuser communication-Part I: Channel inversion and regularization," IEEE Trans. Commun., vol. 53, no. 1, pp. 195-202, Jan. 2005.

[16] J. Arnau-Yanez et al., "Hybrid space-ground processing for highcapacity multi-beam Satellite systems," in Proc. IEEE Global Telecommun. Conf. (GLOBECOM), Dec. 2011, pp. 1-6.

[17] J. Wang and D. P. Palomar, "Worst-case robust MIMO transmission with imperfect channel knowledge," IEEE Trans. Signal Process., vol. 57, no. 8, pp. 3086-3100, Aug. 2009.

[18] D. Palomar, "A unified framework for communications through MIMO channels," Ph.D. dissertation, Tech. Univ. Catalonia, Barcelona, Spain, 2003.

[19] A. Pascual-Iserte, D. P. Palomar, A. I. Perez-Neira, and M. A. Lagunas, "A robust maximin approach for MIMO communications with imperfect channel state information based on convex optimization," IEEE Trans. Signal Process., vol. 54, no. 1, pp. 346-360, Jan. 2006.

[20] D. P. Palomar, J. M. Cioffi, and M. A. Lagunas, "Joint Tx-Rx beamforming design for multicarrier MIMO channels: A unified framework for convex optimization," IEEE Trans. Signal Process., vol. 51, no. 9, pp. 2381-2401, Sep. 2003.

[21] J. Liu, X. Liu, and X. Ma, "First-order perturbation analysis of singular vectors in singular value decomposition," IEEE Trans. Signal Process. vol. 56, no. 7, pp. 3044-3049, Jul. 2008.

[22] M. S. Alouini, S. A. Borgsmiller, and P. G. Steffes, "Channel characterization and modeling for Ka-band very small aperture terminals," Proc. IEEE, vol. 85, no. 6, pp. 981-997, Jun. 1997.

[23] H. Skinnemoen, C. Rigal, A. Yun-Garcia, L. Erup, N. Alagha, and A. Ginesi, "Dvb-rcs2 overview," Int. J. Satellite Commun. Netw., vol. 31, no. 5, pp. 201-217, 2013.

[24] A. Morello and V. Mignone, "DVB-S2: The second generation standard for Satellite broad-band services," Proc. IEEE, vol. 94, no. 1, pp. 210-227, Jan. 2006.

[25] A. W. Marshall and I. Olkin, Inequalities: Theory of Majorization and Its Application. San Diego, CA, USA: Academic, 1979.

[26] C. R. Johnson, "Partitioned and Hadamard product matrix inequalities," J. Res. Nat. Bureau Standards, vol. 83, no. 6, pp. 3044-3049, Nov. 1978.

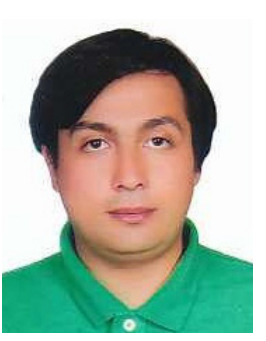

Vahid Joroughi was born in Mianeh, Iran, in 1980 $\mathrm{He}$ received the bachelor's and master's degrees in electrical engineering in 2003 and 2009, respectively. He joined Iran Telecommunication Company (ITC) as a Switching Manager in order to participate in the NEAX project jointly supported by ITC and NEC Company.

From 2012 to 2015, he received the FPU-UPC Grant to conduct the Ph.D. degree jointly with the Center Tecnólogic de Telecomunicacions de Catalunya and Universidad Politécnica de Catalunya, Barcelona. His Ph.D. program was done in the context of different projects provided by European Space Agency on the topic of multibeam satellite communication network. He currently holds a post-doctoral position with the Universidade de Vigo.

His research interest includes satellite communications with special emphasis on MIMO satellite infrastructure, statistical/array signal processing, and cooperative communication.

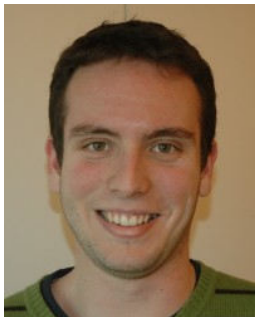

Miguel Ángel Vázquez was born in Palma de Mallorca, Spain, in 1986. He received the Telecommunication Engineering degree from the TelecomBCN Technical University of Catalonia (UPC), the master's degree (Hons.) with a focus on wireless communication in 2012, and the Ph.D. degree in telecommunications from UPC in 2014. His Ph.D. thesis was on beamforming and power control in spectrum sharing systems. He joined the Centre Tecnològic de les Telecomunicacions de Catalunya in 2010. He has been granted a Marie Curie Fellowship in the context of a FP7 European Project (SWAP). He has participated in different EU-funded projects including the FP7, the ARTEMISA, the CELTIC, and the H2020, on the topics of Internet of Things and smart grid, and other industrial contracts with local companies and the European Space Agency. His research interests include statistical and array signal processing, spectrum sharing wireless communications, licensed shared access networks, and satellite communications. He is co-organizing the IEEE ASMSSPSC 2016 Conference and the IEEE S3P Summer School in signal processing for satellite communications.

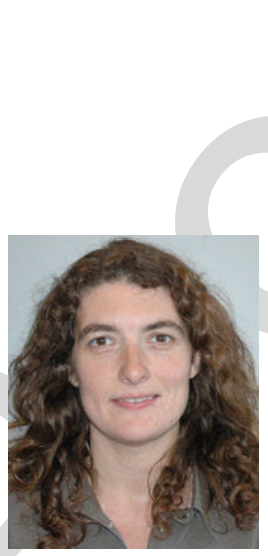

Ana I. Pérez-Neira (S'92-M'95-SM'01) created the UPC Doctoral School in 2011. She was the ViceDirector of Research with UPC from 2010 to 2013 She is currently a Full Professor with the Signa Theory and Communication Department, Technical University of Catalonia (UPC). She is also a Scientific Coordinator with the Centre Tecnològic de les Telecomunicacions de Catalunya. She is also the Coordinator of the European Project SANSA and the Network of Excellence on satellite communications, financed by the European Space Agency: SatnexIV She has been the leader of 20 projects and has participated in over 50 (ten for European Space Agency). She has authored 50 journal papers (20 related to Satcom) and over 200 conference papers (20 invited). She has co-authored four books and five patents (one on Satcom). She has been a guest editor on five special issues. Her research topic is signal processing for communications and currently she is working in multi-antenna and multicarrier signal processing for satellite communications and wireless systems. She was on the Board of Directors of ETSETB (Telecom Barcelona) from 2000 to 2003. Since 2008, she has been a member of the European Signal Processing Association BoD. Since 2010, she has been a member of the IEEE Signal Processing Theory and Methods. She has been the General Chairman of the IWCLD09, the EUSIPCO11, the EW14, and the IWSCS14. She has participated in the organization of the ESA conference 1996 and the SAM04. She will be the General Chair of ASMS16 and the Chair of next IEEE ICASSP20. She is currently an Editor of the IEEE TRANSACTIONS ON Signal PROCESSING and the EURASIP Signal Processing and Advances in Signal Processing.

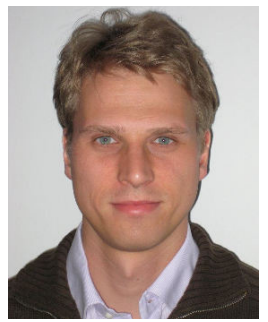

Bertrand Devillers received the degree in electrical engineering and the Ph.D. degree from the Université Catholique de Louvain (UCL), Belgium, in 2004 and 2009, respectively. From 2004 to 2008, he was a Research Fellow of the F.R.S.-FNRS with the Communications and Remote Sensing Laboratory, Digital Communication Group, UCL, Belgium. From 2009 to 2013, he was a Research Associate with the Centre Tecnològic de les Telecomunicacions de Catalunya, Barcelona, Spain. From 2008 to 2009, he was a Research Assistant with UCL, Belgium. His research interests are in communication theory and signal processing for digital terrestrial and satellite communications, multiple input multiple output systems, multicarrier, and cyclically prefixed single carrier modulations, and energy efficiency. 


\section{AUTHOR QUERIES}

\section{AUTHOR PLEASE ANSWER ALL QUERIES}

PLEASE NOTE: We cannot accept new source files as corrections for your paper. If possible, please annotate the PDF proof we have sent you with your corrections and upload it via the Author Gateway. Alternatively, you may send us your corrections in list format. You may also upload revised graphics via the Author Gateway.

AQ:1 = Please be advised that per instructions from the Communications Society this proof was formatted in Times Roman font and therefore some of the fonts will appear different from the fonts in your originally submitted manuscript. For instance, the math calligraphy font may appear different due to usage of the usepackage[mathcal]euscript. We are no longer permitted to use Computer Modern fonts.

AQ:2 = Please provide the postal codes for "Universidade de Vigo, Centre Tecnològic de les

Telecomunicacions de Catalunya, and Universitat Politècnica de Catalunya."

AQ:3 = Please provide the current affiliation of "Bertrand Devillers."

AQ:4 = Note that if you require corrections/changes to tables or figures, you must supply the revised files, as these items are not edited for you.

AQ:5 = Please confirm the volume no. for ref. [11].

AQ:6 = Please provide the department name for ref. [18].

AQ:7 = Please confirm whether the edits made in the sentence "He joined... satellite communication network." are OK. 


\title{
Onboard Beam Generation for Multibeam Satellite Systems
}

\author{
Vahid Joroughi, Miguel Ángel Vázquez, Ana I. Pérez-Neira, Senior Member, IEEE, and Bertrand Devillers
}

\begin{abstract}
This paper aims at designing an onboard beam generation process for a hybrid onboard on-ground multibeam satellite architecture. The proposed method offers a good tradeoff between total throughput and feeder link bandwidth requirements compared with pure on-ground systems. Full frequency reuse among beams is considered, and the beamforming at the satellite is designed for supporting interference mitigation techniques. In addition, in order to reduce the payload cost and complexity, this onboard beamforming is assumed to be constant and the same for forward and return link transmissions so that the same array-fed reflector can be used for forward and return links, leading to a substantial reduction of the payload mass. To meet all these requirements, a novel robust minimum mean square error optimization is conceived. The benefits of the considered scheme are evaluated with respect to the current approaches both analytically and numerically. Indeed, we show that with the DVB-RCS and DVB-S2 standards, our proposal allows increasing the total throughput within a range between $6 \%$ and $15 \%$ with respect to other onboard processing techniques in the return and forward link, respectively.
\end{abstract}

Index Terms-Multibeam satellite systems, on-board beam processing, linear precoding, DVB-S2, DVB-RCS. HE increasing demand for fixed broadband data services is an opportunity for satellite industries to target new markets apart from the well-known current ones (i.e. broadcast broadband, emergency communications, ...). In order to cope with higher data traffic demands, satellite system designers are looking for advanced satellite communication architectures. In this context, the use of multiple beams has recently received a lot of attention as a key enabler of next generation high throughput satellite systems. These systems rely on employing a large number of beams instead of a single (global) beam

Manuscript received May 11, 2015; revised November 11, 2015, May 11, 2016, and October 7, 2016; accepted March 8, 2017. This work was supported in part by the Spanish Ministry of Economy and Competitiveness (ELISA) under Project TEC2014-59255-C3-1-R and in part by the Catalan Government under Grant 2014SGR1567. This work was presented at the IEEE Globecom 2013. The associate editor coordinating the review of this paper and approving it for publication was L. Liu.

V. Joroughi is with the Universidade de Vigo, Vigo, Spain (e-mail: vahid.joroughi@gts.uvigo.es).

M. Á. Vázquez is with the Centre Tecnològic de les Telecomunicacions de Catalunya, Barcelona, Spain (e-mail: mavazquez@cttc.es).

A. I. Pérez-Neira is with the Centre Tecnològic de les Telecomunicacions de Catalunya, Barcelona, Spain, and also with the Universitat Politècnica de Catalunya, Barcelona, Spain (e-mail: ana.isabel.perez@upc.edu).

B. Devillers was with the Centre Tecnològic de les Telecomunicacions de Catalunya, Barcelona, Spain (e-mail: bertrand.devillers@gmail.com).

Color versions of one or more of the figures in this paper are available online at http://ieeexplore.ieee.org.

Digital Object Identifier 10.1109/TWC.2017.2687924 in the coverage area. This is beneficial since each beam can have a larger antenna gain-to-noise temperature than in the single beam case and the available spectrum can be reused among spatially separated beams. Furthermore, whenever the satellite systems delivers broadband unicast (i.e. a single user per beam is served) or multicast (i.e. multiple user per beam are served) interactive traffic, the multibeam architecture can support different modulations and code rates for each user depending on the user link quality, leading to a high increase of the overall system throughput.

Nowadays, the system designers target the Terabit multibeam satellite system, i.e. a satellite system offering a Terabit per second capacity. In fact, the goal is to increase the overall spectral efficiency while keeping the payload complexity affordable. One of the main challenges of Terabit satellite systems is how to deal with the large spectral demands of the feeder link (i.e. the bidirectional communication link between the satellite and the service provider), whose bandwidth requirements increase exponentially as it aggregates the traffic of all users, while keeping a full frequency reuse allocation. Recently, some techniques have been proposed in order to optimize the feeder link spectrum resources. Indeed, there is a current tendency for moving the feeder from the Ka band to the $\mathrm{Q} / \mathrm{V}$ band, where there are larger available bandwidths [1]. Unfortunately, in these frequencies the fading is extremely large and more advanced transmitting diversity techniques are needed.

Another option is the use of multiple gateways, which might be adequate in order to reduce the feeder link spectral requirements as they can be equipped with very directive antennas and exploit the spatial diversity while sharing all available spectrum [2], [3]. Nevertheless, the deployment of several gateways increases the cost of the system and; moreover, the interference mitigation techniques result in certain performance degradation [4], [5]. This is due to the fact that the processing must be separated in isolated processing units.

In contrast to the aforementioned satellite architectures, this paper focuses on the hybrid on-board on-ground processing scheme. This promising solution keeps certain operations in the payload so that the amount of required signals from the feeder link are severely reduced. In this way, the satellite does act in transparent mode and it analogically processes the signals, leading to a high reduction of the feeder link bandwidth requirements. Specifically, while the full on-ground beamforming requires a feeder link bandwidth of

$$
B_{\text {feeder link on-ground }}=N B_{\text {beam }} \text {, }
$$


where $N$ is the number of feed elements ${ }^{1}$ and $B_{\text {beam }}$ is the the total available bandwidth that the users employ; the hybrid on-board on-ground processing only requires

$$
B_{\text {feeder link hybrid }}=K B_{\text {beam }},
$$

where $K$ is the number of beams. As a result, the feeder link bandwidth is reduced since for this work we will consider multiple-feed-per-beam architecture where $N>K$. Note that, in contrast to single-feed-per-beam architectures $(N=K)$, in multiple-feed-per-beam architectures beamforming scan losses are negligible [6]. In addition, multiplefeed-per-beam architecture allows a single on-board reflector to emit beams since single-feed-per-beam typically requires multiple antenna reflectors for a contiguous coverage. A more detailed description of the beam process and its feeder link requirements is presented in [7] and [8].

Apart from the feeder link challenge, multibeam satellite systems require a large capacity in the access network. As a matter of fact, in the generated radiation pattern on Earth, adjacent beams create high levels of interference and, therefore, a carefully planned power and frequency reuse among beams must be employed to cope with this increased level of interference. Consequently, beams with adjacent footprint currently operate in different frequency bands or polarizations. In this context, an essential parameter is the number of colors $N_{c}$ in the frequency reuse pattern, which we define as the cardinality of the set of disjoint frequency bands and polarizations used on the cluster of beam footprints which define the coverage area $\left(N_{c} \geq 1\right)$. In fact, the lower the number of $N_{c}$, the higher the overall system bandwidth will be and the higher the interference power levels will be generated.

In order to increase the available bandwidth yet maintain a low multiuser interference, a promising technique is to use full frequency reuse pattern $\left(N_{c}=1\right)$ and resort to interference mitigation techniques. In this way, signals can be precoded and detected before being transmitted and received in order to reduce inter-beam interference [9]. As a result, a considerable improvement of the achievable spectral efficiency can be obtained. To this end, more advanced interference mitigation techniques as precoding in the forward link and multiuser detection or filtering in the return link have been considered in past studies of the European Space Agency (ESA) [9], [10].

Since interference mitigation techniques require large computational resources, they must be carried out on ground. Indeed, larger efficiencies are obtained if not only the precoding and detection are done on ground, but also the beam generation process, as more flexible processing units are available. In other words, if the beamforming is kept fixed on the payload, there is a performance loss compared to the spectral efficiencies obtained by on ground beamforming [11], [12]. However, if the satellite does not perform any beam processing, the feeder link needs a large amount of spectral resources in order to transmit all the user signals. In addition, centralizing signal processing mechanisms on-ground requires a phase calibration loop between satellite and ground segment. Consequently, even though certain degradation is expected

\footnotetext{
${ }^{1}$ The input signals of the antenna array feed assembly located in the payload.
}

with respect to the on-ground operation (i.e. beam generation, precoding and detection are done in the terrestrial segment), in the present work we propose to optimize the on-board beam generation process so that the achievable rates do not severely decrease due to the on-board beam generation and the feeder link traffic is kept low.

Concretely, this paper focuses on obtaining an optimal onboard beam generation when linear minimum mean square error (LMMSE) precoding technique in the forward link and LMMSE detection procedure in the return link are used as interference mitigation techniques. This study foresees the presence of a non-channel-adaptive (fixed) on-board beam processing scheme in order to keep payload complexity low. Thus, the problem becomes more difficult in the presence of this fixed process in the payload. In order to deal with this problem, we use a robust optimization framework so that a fixed beam generation can be obtained despite user link channel variation.

Furthermore, the designs for both the forward and return links results the same, which makes it appropriate for the future multibeam satellite systems since it is expected that the same reflector is employed at the return and forward links leading to a substantial cost and mass reduction of the payload. Note that the variability of the channel is due to the change of position of the users in consecutive time instants and atmospheric fading. Numerical simulations show the benefit of our method, which in some scenarios can increase the spectral efficiency over the $6 \%$ and $15 \%$ for return and forward links, respectively, if the DVB-S2 and DVB-RCS modulation and coding parameters (modcods) are used.

To the best of the authors knowledge, this is the first time the problem of on-board beam generation process is treated not only in the forward but also in the return link. In contrast to our preliminar work [13], where only the forward link was examined, in this paper we focus our attention to the joint forward and return link optimization. In addition, a novel and better robust design is presented based on a more complete description of the channel perturbations. This new scheme is conceived considering a first order perturbation approach. Finally, several detailed evaluations are presented that validate our contribution in detail.

To sum up, the paper contributions are:

- We propose an on-board beamforming scheme that results the same for forward and return links leading to a substantial reduction of the satellite mass and cost.

- This on board processing considers that precoding and filtering is used so that larger gains are obtained with respect to the schemes that only consider the coverage area.

- In addition, this on board beamforming can keep the feeder link bandwidth requirements low yet preserving substantial gains over the coverage area with respect to full on ground techniques.

- The proposed technique is robust and invariant to channel variations and a novel perturbation analysis is performed.

- Numerical simulations are performed in a close-to-real scenario considering a real deployment which leads to an adequate validation. 
The rest of the paper is organized as follows: Section II presents the signal model. A brief introduction of the beam generation process and the problem characteristics are described in section III. Section IV presents a novel fixed onboard beam generation process. Section V presents a novel robust scheme based on a first order perturbation analysis. Section VI contains a summary of the simulation results, and eventually the conclusions are given in section VI.

Notation: Throughout this paper, the following notations are adopted. Boldface upper-case letters denote matrices and boldface lower-case letters refer to column vectors. $(.)^{H}$, $(.)^{T},(.)^{*}$ and $(.)^{+}$denote a Hermitian transpose, transpose, conjugate and diagonal (with positive diagonal elements ) matrix, respectively. $\mathbf{I}_{N}$ builds $N \times N$ identity matrix and $\mathbf{0}_{K \times N}$ refers to an all-zero matrix of size $K \times N$. If $\mathbf{A}$ is a $N \times N$ matrix, $\mathbf{A}_{1: K}$ refers to taking the $K$ first rows of the matrix A. $(\mathbf{X})_{i j}$ represents the $(i$-th, $j$-th) element of matrix $\mathbf{X}$. If $\mathbf{B}$ is a $N \times N$ matrix, $\mathbf{A} \leq \mathbf{B}$ implies $\mathbf{A}-\mathbf{B}$ is semidefinite negative. A matrix $\mathbf{M}$ is definite negative if the real part of $\mathbf{z}^{H} \mathbf{M z} \leq 0$ for any non-zero $\mathbf{z}$. $\mathbf{a} \prec \mathbf{b}$ means vector a majorizes vector $\mathbf{b}$. Finally, E $\{$.$\} and \|$.$\| refer to the$ expected value operator and the Frobenius norm, respectively. The operator o corresponds to the Hadamart product which is a componentwise product.

\section{Signal MODEL}

Let us consider a multibeam satellite communication system, where a single geosynchronous satellite with multibeam coverage provides fixed broadband services to a large set of users, typically operating in Ka-band, although $\mathrm{L}$ and $\mathrm{S}$ band could be considered depending on the scenario. To this end, the satellite is equipped with an array fed reflector antenna whose number of feeds is denoted by $N$. The coverage area is divided into $K$ beams, with

$$
K<N,
$$

and the users are assumed to be uniformly distributed within the beams. By employing a time division multiplexing access (TDMA) scheme, at each time instant the gateway is serving a total of $K$ single antenna users (i.e. exactly one user per beam), and it is transmitting (receiving) information to (from) the same number of the users through the satellite in the forward (return) link. Note that in return link satellite communications generally operate in a multi-frequency TDMA (MF-TDMA) so that different users of the same beam might be allocated to different sub-bands. For the sake of simplicity and without loss of generality, the rest of the paper considers TDMA for the return link. Remarkably, the conceived technique can be accommodated to the multi-band communication by replicating the linear processing at each band due to the frequency flatness of the channel response.

The satellite is assumed to linearly convert a set of $N$ onboard feed signals into the $K$ feeder link signals which are transmitted to the gateway in a frequency multiplexed fashion. Reciprocally, in the forward link, the same linear processing strategy is used to construct the $N$ feed signals from the $K$ feeder link signals.

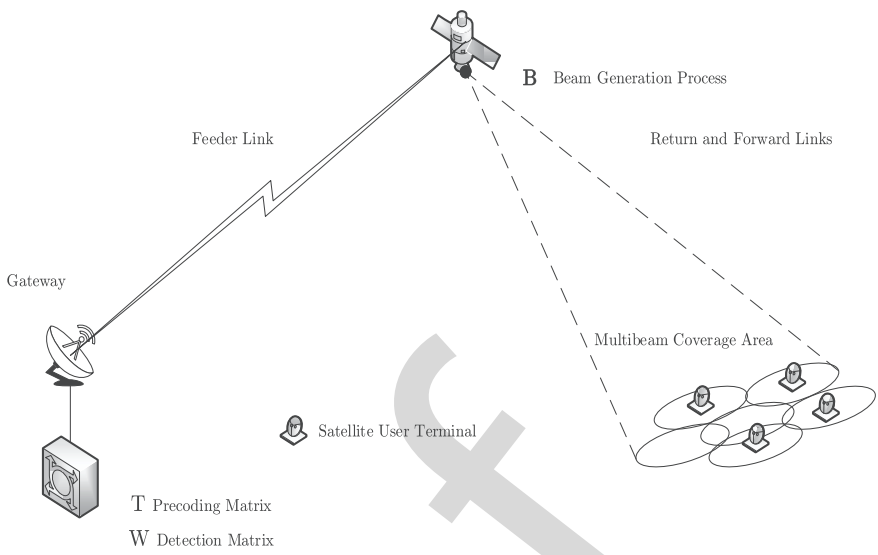

Fig. 1. Multibeam satellite system with on-board beam generation process. The precoding and detection procedures are done on ground. On the contrary, the beam generation process is carried out at the payload and it is assumed to be constant and the same for forward and return links.

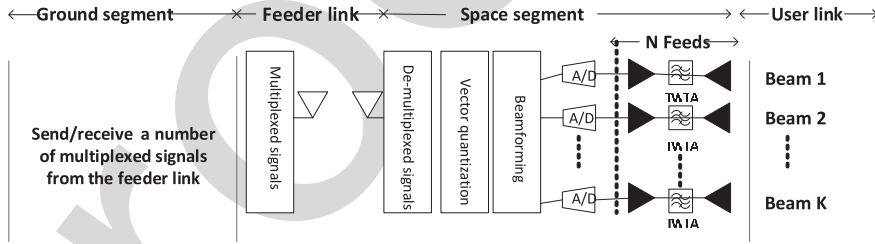

Feeder link: transmits the signals received by gateway to the satellite in a multiplexed fashion Feeds: each feed is shared by multiple beams making TWTAs always operating in multicarrier mode

Fig. 2. Block diagram of an hybrid on-board on-ground multibeam satellite architecture.

Moreover, since a high throughput system is targeted, full frequency reuse among beams is assumed so that all beams can share the same frequency resources. The user link is the communication bottleneck of the whole system. The feeder link is assumed perfectly calibrated and noiseless. Figures 1 and 2 summarize the transmission block diagram.

In the following, the mathematical expressions of the signal model in both the return and forward links are described.

\section{A. Return Link}

As stated above, $K$ denotes the number of users and $N$ is the number of on-board feeds. Then, the corresponding received signal at the gateway can be modelled as

$$
\mathbf{y}_{\mathrm{RL}}=\sqrt{\beta} \mathbf{B H s}+\mathbf{B n},
$$

where $\mathbf{y}_{R L}=\left[y_{R L, 1}, \ldots, y_{R L, K}\right]^{T}$ is a $K \times 1$ vector containing the stack of received signals at the gateway. The $K \times 1$ vector $\mathbf{S}$ is the stack of the transmitted independent signals by all users such that $\mathrm{E}\left\{\mathbf{s s}^{H}\right\}=\mathbf{I}_{K}$. Note that, throughout the paper the subscript $\mathrm{RL}$ is used to refer the return link while $\mathrm{FL}$ will denote the forward link. The constant $\beta$ denotes Equivalent Isotropic Radiated Power (EIRP), which is referred to the user terminal transmit power and we assume to be the same for all the users.

In order to radiate the multibeam pattern, the satellite payload is equipped with a smart antenna system (generally an array fed reflector) coined as on-board beam generation 
process. This system constructs the beam pattern for transmitting and receiving data from the coverage area. Mathematically, the effect of this beamforming appears as the rectangular $K \times N$ fat matrix $\mathbf{B}$.

The $N \times 1$ vector $\mathbf{n}$ accounts for the zero mean Additive White Gaussian Noise (AWGN). We assume unit variance Gaussian noise samples such that

$$
\mathrm{E}\left\{\mathbf{B n n}^{H} \mathbf{B}^{H}\right\}=\mathbf{B B}^{H} .
$$

For radio-frequency design convenience, we will assume that B is orthonormal so that the feed signals are decoupled at the payload $\left(\mathbf{B B}^{H}=\mathbf{I}_{K}\right)$. Matrix $\mathbf{H}$ is the overall $N \times K$ user link channel matrix whose element $\mathrm{h}_{i j}$ presents the aggregate gain of the link between the $i$-th satellite feed and the $j$-th user (in the $j$-th beam). This channel can be decomposed as follows:

$$
\mathbf{H}=\mathbf{G D},
$$

where:

- $\mathbf{G}$ is a $N \times K$ matrix that models the feed radiation pattern, the on-board attenuation and path losses. It is responsible for the interference among users. We assume the elements of $\mathbf{G}$ are normalized so that they have unit variance.

- D is assumed to be a $K \times K$ diagonal matrix which takes into account the atmospheric fading in the user link.

Note that (k,n)-th entry of the feed radiation pattern matrix $\mathbf{G}$ can be described as follows

$$
(G)_{k, n}=\frac{G_{R} a_{k n}}{4 \pi \frac{d_{k}}{\lambda} \sqrt{K_{B} T_{R} B_{W}}},
$$

with $d_{k}$ the distance between the $k$-th user terminal and the satellite. $\lambda$ is the carrier wavelength, $K_{B}$ is the Boltzmann constant, $B_{W}$ is the carrier bandwidth, $G_{R}^{2}$ the user terminal receive antenna gain, and $T_{R}$ the receiver noise temperature. The term $a_{k n}$ refers to the gain from the $n$-th feed to the $k$-th user. It is important to mention that the $\mathbf{G}$ matrix has been normalized to the receiver noise term. The reader can refer to [12] for a more detailed description of the channel model.

\section{B. Forward Link}

Analogously to the return link, the signal model of the forward link becomes

$$
\mathbf{y}_{\mathrm{FL}}=\gamma \mathbf{H}^{T} \mathbf{B}^{T} \mathbf{x}+\mathbf{w},
$$

where $K \times 1$ vector $\mathbf{y}_{\mathrm{FL}}$ is the stack of received signals at each user terminal, and $\mathbf{x}$ is a $K \times 1$ vector that contains the stack of transmitted symbols. Remarkably, in general wireless communication systems, the channel reciprocity does not hold as uplink and downlink operate in disjoint frequency bands. However, considering our channel modelling, the channel matrix in the forward link differs from the return link in the path loss, feed gain and atmospheric fading. As a result, a scaling factor $\gamma$ can model the different frequency operations.

Similarly as in the return link, $\mathbf{w}$ is a $K \times 1$ vector that represents the independent and identically distributed zero mean Gaussian random noise with unit variance such that

$$
E\left\{\mathbf{w} \mathbf{w}^{H}\right\}=\mathbf{I}_{K} .
$$

Evidently, B does not influence in the forward link noise covariance matrix. We assume the following average available power constraint:

$$
\operatorname{trace}\left(\mathbf{x} \mathbf{x}^{H}\right) \leq P_{F L},
$$

where $P_{\mathrm{FL}}$ denotes the total transmit power in the forward link. Note that the transmit power constraint is set without considering the beam generation process $\mathbf{B}$. This is because the power allocation mechanism is located before the array fed reflector system. In addition, it is assumed that the feeds can share the available transmit power. This can be implemented with flexible travelling wave tube amplifiers jointly with multiport power amplifiers as described in [14].

Now, we proceed to jointly optimize matrix $\mathbf{B}$ so that the overall system performance is improved. It is important to remark that $\mathbf{B}$ must be the same for both the optimization of the return and forward links in order to reduce the payload cost. In addition, this matrix needs to be constant in order to keep the payload complexity low and minimize the feeder link spectral resources.

\section{PROBLEM Formulation}

Let us assume that the gateway has perfect Channel State Information (CSI) and uses LMMSE as described in [15] for precoding in the forward link and LMMSE filtering for multiuser detection in the return link. These techniques have been pointed out as efficient methods due to both its interference rejection capabilities and fairness among beams while preserving a low computational complexity [16].

This work resorts to the minimization of the trace of the MSE matrix both at the forward and return links that results from the use of LMMSE precoding and detection. Let us briefly outline the overall mathematical derivation:

1) First, the MSE matrix of the return link is computed assuming LMMSE detection.

2) Second, the MSE matrix of the forward link is computed assuming LMMSE precoding.

3) Third, an upper bound of the MSE minimization in the return link is presented.

4) Finally, a novel robust beam generation process in the return link, which considers the aforementioned upper bound is obtained. For the forward link case, the optimal design yields to the same solution as it is described.

Remarkably, the design of the optimal $\mathbf{B}$ is imposed to be non channel dependent. We show that the optimal $\mathbf{B}$ in the forward and return links results to be the same; thus, fulfilling one of the constraints of the system.

\section{A. Return Link}

As a first step, let us define $\mathbf{W}^{H}$ as the LMMSE filter that detects $K$ received signals at the gateway such that $\hat{\mathbf{s}}=\mathbf{W}^{H} \mathbf{y}_{\mathrm{RL}} ;$ composed by $\hat{\mathrm{s}}_{i}$ which denotes the $i$-th element of the detected signal (for $i$-the user) in the gateway. In this context, the MSE of $i$-th user is achieved as follows

$$
\mathrm{MSE}_{\mathrm{RL}, i}=\mathrm{E}\left\{\left|\mathrm{s}_{i}-\hat{\mathrm{s}}_{i}\right|^{2}\right\},
$$


where $\mathrm{s}_{i}$ represents the $i$-th element of transmit signal vector (for $i$-the user) for a total of $K$ users such that $\mathbf{s}=$ $\left(s_{1}, \ldots, s_{K}\right)^{T}$.

It is well known that the mathematical expression of LMMSE filter becomes

$$
\mathbf{W}^{H}=\left(\mathbf{I}_{K}+\beta \mathbf{H}^{H} \mathbf{B}^{H} \mathbf{B H}\right)^{-1} \mathbf{H}^{H} \mathbf{B}^{H},
$$

and the MSE matrix after the use of this filter is

$$
\mathbf{M S E}_{\mathrm{RL}}=\left(\mathbf{I}_{K}+\beta \mathbf{H}^{H} \mathbf{B}^{H}\left(\mathbf{B B}{ }^{H}\right)^{-1} \mathbf{B H}\right)^{-1} .
$$

Without loss of generality, we restrict $\mathbf{B}$ to be orthonormal such that $\mathbf{B B}^{H}=\mathbf{I}_{K}$. The sum of MSE in the return link is defined as

$$
\mathrm{SMSE}_{\mathrm{RL}}=\operatorname{trace}\left(\left(\mathbf{I}_{K}+\beta \mathbf{H}^{H} \mathbf{B}^{H} \mathbf{B H}\right)^{-1}\right) .
$$

Now, let us assume for a moment that $\mathbf{B}$ can be channel adaptive (i.e the payload can modify $\mathbf{B}$ depending on the channel variations). Then, the corresponding problem is formulated as

$$
\begin{aligned}
& \min _{\mathbf{B}} \operatorname{trace}\left(\left(\mathbf{I}_{K}+\beta \mathbf{H}^{H} \mathbf{B}^{H} \mathbf{B} \mathbf{H}\right)^{-1}\right) \\
& \text { s.t. } \mathbf{B B}^{H}=\mathbf{I}_{K} .
\end{aligned}
$$

It is important to remark that the authors in [11] showed that the presence of $\mathbf{B}$ increases the $\mathrm{SMSE}_{\mathrm{RL}}$ in the gateway. Mathematically,

$$
\begin{aligned}
& \operatorname{trace}\left(\left(\mathbf{I}_{K}+\beta \mathbf{H}^{H} \mathbf{B}^{H} \mathbf{B} \mathbf{H}\right)^{-1}\right) \\
& \geq \operatorname{trace}\left(\left(\mathbf{I}_{K}+\beta \mathbf{H}^{H} \mathbf{H}\right)^{-1}\right) .
\end{aligned}
$$

Indeed, in [11] it was shown that with the following Singular Value Decomposition (SVD) of the channel $\mathbf{H}=\mathbf{U} \boldsymbol{\Phi} \mathbf{V}^{H}$, an optimal design of $\mathbf{B}$ can be worked out as

$$
\mathbf{B}=\mathbf{U}_{1: K}^{H},
$$

where $\mathbf{U}_{1: K}^{H}$ denotes the $K$ first rows of the matrix $\mathbf{U}^{H}$. In fact, it can be easily seen that this particular solution reaches equality in (16) and; thus, minimizes the $\mathrm{SMSE}_{\mathrm{RL}}$.

In the present work, $\mathbf{B}$ is assumed to be non-channel adaptive, therefore, the design of $\mathbf{B}$ in (17) cannot be considered. Even though the channel appears to be variable at each realization, we aim at finding the best possible non-channel adaptive design of $\mathbf{B}$. In this context, let us decompose the channel as follows

$$
\mathbf{H} \triangleq \overline{\mathbf{H}}+\boldsymbol{\Delta},
$$

where:

- $\overline{\mathbf{H}}$ represents the mean value of the channel.

- $\Delta$ models the difference between the actual value of the channel and its mean. It indicates the variability of the channel in consecutive time instants as already explained in section I.

We assume that the actual channel $\mathbf{H}$ lies in the neighborhood of a nominal channel $\overline{\mathbf{H}}$ that is known to the gateway.
In particular, we consider that $\mathbf{H}$ belongs to the uncertainty ${ }_{422}$ region $\mathcal{H} \triangleq\{\mathbf{H}:\|\mathbf{H}-\overline{\mathbf{H}}\| \leq \alpha\}$ which is an sphere centered at $\overline{\mathbf{H}}$ with the radius $\alpha$.

Interestingly, the channel model in (18) resembles the modeling of a MIMO system with imperfect CSI at the transmitter which has been solved as a worst case optimization problem in [17]-[19]. With this perspective for the return link, the worst case robust design is proposed, which leads to a maximin or minimax formulation:

$$
\begin{aligned}
& \min _{\mathbf{B}} \max _{\Delta} \operatorname{trace}\left(\left(\mathbf{I}_{K}+\beta \mathbf{H}^{H} \mathbf{B}^{H} \mathbf{B} \mathbf{H}\right)^{-1}\right) \\
& \text { s.t. } \mathbf{B B}^{H}=\mathbf{I}_{K} .
\end{aligned}
$$

Prior to obtaining the solution of (19), let us focus on the forward link optimization problem, which is similarly derived.

\section{B. Forward Link}

In the forward link, the zero forcing precoding with a regularized inversion is assumed [15]. In this case, the linear precoding is expressed as

$$
\mathbf{x}=\mathbf{T c},
$$

where $\mathbf{T}$ is the $K \times K$ precoding matrix at the gateway and c is the $K \times 1$ transmit symbol vector at all feeds such that $\mathrm{E}\left\{\mathbf{c c}^{H}\right\}=\mathbf{I}_{K}$. In this context, the corresponding precoding matrix $\mathbf{T}$ is expressed as

$$
\mathbf{T}=\gamma \sqrt{\rho} \mathbf{B}^{*} \mathbf{H}^{*}\left(\frac{K}{P_{\mathrm{FL}}} \mathbf{I}_{K}+\gamma^{2} \mathbf{H}^{T} \mathbf{B}^{T} \mathbf{B}^{*} \mathbf{H}^{*}\right)^{-1},
$$

where the value of the constant $\rho$ has to comply with the forward link power constraint as follows

$$
\operatorname{trace}\left(\mathbf{T T}^{H}\right) \leq P_{\mathrm{FL}} .
$$

It is important to remark that in order to properly decode the transmitted symbols, the receivers shall know $\rho$ a prior so that the transmitter shall share this value to all receivers jointly with the transmitted frame. This can be done with during the pilot symbol transmission where precoding is not applied.

This particular kind of precoder is used to find an optimal balance between achieving signal gain and limiting the multiuser interference. Similar to the return link, $\mathrm{MSE}_{\mathrm{FL}, i}$ is defined as

$$
\operatorname{MSE}_{F L, i}=\mathrm{E}\left\{\left|\mathrm{c}_{i}-\hat{\mathrm{c}}_{i}\right|^{2}\right\},
$$

where $\mathrm{MSE}_{\mathrm{FL}, i}$ refers to the MSE received by $i$-th user. Similarly, $\mathbf{c}=\left(c_{1}, \ldots, c_{K}\right)^{T}$ and $\hat{\mathbf{c}}=(\sqrt{\rho})^{-1} \mathbf{y}_{\mathrm{FL}}=\left(\hat{c}_{1}, \ldots, \hat{c}_{K}\right)^{T}$ are the transmitted and received signals for $K$ users, respectively. In this context, $c_{i}$ represents the transmitted signal for $i$-the user and $\hat{\mathrm{c}}_{i}$ denotes the signal received by user $i$-th. The MSE matrix in the forward link can be calculated as follows

$$
\mathbf{M S E}_{\mathrm{FL}}=\mathrm{E}\left\{\left((\sqrt{\rho})^{-1} \mathbf{y}_{\mathrm{FL}}-\mathbf{c}\right)\left((\sqrt{\rho})^{-1} \mathbf{y}_{\mathrm{FL}}-\mathbf{c}\right)^{H}\right\},
$$


which can be rewritten as

$$
\begin{aligned}
\mathbf{M S E}_{\mathrm{FL}}=\frac{K}{P_{\mathrm{FL}}}( & \left(\gamma^{2} \mathbf{H}^{T} \mathbf{B}^{T} \mathbf{B}^{*} \mathbf{B}^{T} \mathbf{B}^{*} \mathbf{H}^{*}+\frac{K}{P_{\mathrm{FL}}} \mathbf{I}_{K}\right) \\
& \left.\times\left(\gamma^{2} \mathbf{H}^{T} \mathbf{B}^{T} \mathbf{B}^{*} \mathbf{H}^{*}+\frac{K}{P_{\mathrm{FL}}} \mathbf{I}_{K}\right)^{-2}\right)
\end{aligned}
$$

As in the return link, we concentrate our efforts to minimize the sum of MSE, this is

$$
\mathrm{SMSE}_{\mathrm{FL}}=\operatorname{trace}\left(\mathbf{M S E}_{\mathrm{FL}}\right),
$$

where, recalling that $\mathbf{B B}^{H}=\mathbf{I}_{K}$ and we consider the following property, $\operatorname{trace}(\mathbf{A})=\operatorname{trace}\left(\mathbf{A}^{T}\right)$ where $\mathbf{A}$ is a square matrix, then we have that

$$
\operatorname{SMSE}_{F L}=\frac{K}{P_{\mathrm{FL}}} \operatorname{trace}\left(\left(\gamma^{2} \mathbf{H}^{H} \mathbf{B}^{H} \mathbf{B H}+\frac{K}{P_{\mathrm{FL}}} \mathbf{I}_{K}\right)^{-1}\right) .
$$

The worst case optimization problem thanks to the channel decomposition in (18) can be formulated as follows

$$
\begin{aligned}
& \min _{\mathbf{B}} \max _{\Delta} \operatorname{trace}\left(\left(\gamma^{2} \mathbf{H}^{H} \mathbf{B}^{H} \mathbf{B H}+\frac{K}{P_{\mathrm{FL}}} \mathbf{I}_{K}\right)^{-1}\right) \\
& \text { s.t. } \mathbf{B B}{ }^{H}=\mathbf{I}_{K} .
\end{aligned}
$$

Note that the return link optimization (19) and the forward link one (28) are the same except for a scalar value. In next section we show that both lead to the same optimal design; thus confirming a natural uplink downlink physical duality.

\section{B OptimizATION}

This section tackles with the main objective of this paper. An optimally designed $\mathbf{B}$ for problems (19) and (28) is presented. Two main steps are followed. The first step provides a brief description of an upper bound for the SMSE. The second step proposes a design for $\mathbf{B}$ such that it minimizes the proposed SMSE upper-bound obtained in the first step. The design is done for the return link and extended to the forward link.

Prior to presenting the optimal design, we need to introduce the next lemma.

Lemma 1: Assuming an arbitrary square matrix $\mathbf{A}$, the next equation holds

$$
\operatorname{trace}\left(\left(\mathbf{I}_{K}+\mathbf{A A}^{H}\right)^{-1}\right)=\operatorname{trace}\left(\left(\mathbf{I}_{K}+\mathbf{A}^{H} \mathbf{A}\right)^{-1}\right) .
$$

Proof: It is a direct consequence of inversion matrix lemma.

By considering $\mathbf{A}=\sqrt{\beta} \mathbf{B H}$, the $\mathrm{SMSE}_{\mathrm{RL}}$ in problem (19) can be rewritten as

$$
\operatorname{trace}\left(\left(\mathbf{I}_{K}+\beta \mathbf{B Z Z B} \mathbf{B}^{H}\right)^{-1}\right)
$$

where $\mathbf{Z}=\mathbf{H} \mathbf{H}^{H}=\overline{\mathbf{H}} \overline{\mathbf{H}}^{H}+\overline{\mathbf{H}} \boldsymbol{\Delta}^{H}+\boldsymbol{\Delta} \overline{\mathbf{H}}^{H}+\boldsymbol{\Delta} \boldsymbol{\Delta}^{H}$ is a $N \times N$ matrix. We propose an upper bound of $\mathrm{SMSE}_{\mathrm{RL}}$ as follows

Theorem 1: The $S M S E_{R L}$ is upper bounded by

$$
\operatorname{trace}\left(\left(\mathbf{I}_{K}+\beta \mathbf{B Z Z B} \mathbf{B}^{H}\right)^{-1}\right) \leq \operatorname{trace}\left(\left(\mathbf{I}_{K}+\beta \mathbf{B} \breve{\mathbf{Z}} \mathbf{B}^{H}\right)^{-1}\right)
$$

where

$$
\breve{\mathbf{Z}} \triangleq \overline{\mathbf{U}}\left(\overline{\boldsymbol{\Sigma}}-\epsilon_{H} \mathbf{I}_{N}\right)^{+} \overline{\mathbf{U}}^{H},
$$

so that $\overline{\mathbf{H}} \overline{\mathbf{H}}^{H}=\overline{\mathbf{U}} \overline{\mathbf{\Sigma}} \overline{\mathbf{U}}^{H}$ is the eigen-decomposition of matrix $\overline{\mathbf{H}} \overline{\mathbf{H}}^{H}$. The scalar value $\epsilon_{H}$ is defined as

$$
\epsilon_{H} \triangleq 2 \alpha \delta_{\max }(\overline{\mathbf{H}})
$$

where $\delta_{\max }(\mathbf{C})$ denotes the maximum singular value of $\mathbf{C}$ matrix.

Proof: See [18, Sec.7.3.1].

As a result, a worst-case $S_{M S E}$ can be obtained in practice by using the lower bound $\breve{\mathbf{Z}}$ in lieu of $\mathbf{Z}$. However, it is important to mention that some values of $\alpha$ lead to unfeasible $\mathrm{MSE}_{\mathrm{RL}}$ solutions, that is, for a large value of $\alpha$ the matrix (32) might become low rank since ( $)^{+}$operator delivers 0 whenever the diagonal entry is non positive. Due to that, the value of $\alpha$ has to be checked and, if necessary, decreased so that

$$
\operatorname{rank}(\breve{\mathbf{Z}})=K \text {. }
$$

In order to obtain a robust design, the target is to minimize the proposed upper-bound of $\mathrm{SMSE}_{R L}$ in (31) instead of (19). In this case, the corresponding problem is formulated as

$$
\begin{aligned}
& \min _{\mathbf{B}} \operatorname{trace}\left(\left(\mathbf{I}_{K}+\beta \mathbf{B} \breve{\mathbf{Z}} \mathbf{B}^{H}\right)^{-1}\right) \\
& \text { s.t. } \mathbf{B} \mathbf{B}^{H}=\mathbf{I}_{K} .
\end{aligned}
$$

The solution to this optimization problem is described in the next theorem.

Theorem 2: The upper bound of SMSE is minimized if $\mathbf{B}$ is selected as the first $K$ rows of the matrix $\overline{\mathbf{U}}^{H}$, that is

$$
\mathbf{B}^{\star}=\overline{\mathbf{U}}_{1: K}^{H},
$$

where $\mathbf{B}^{\star}$ denotes the optimal design of $\mathbf{B}$.

Proof: See Appendix A.

Remark: It is important to mention that the derivation of theorem 2 differs to [20, Th. 1]. The main difference consists of the constraint since in [20] a total power constraint is considered

$$
\operatorname{trace}\left(\mathbf{B B}^{H}\right) \leq P_{\mathrm{FL}},
$$

where as this paper assumes

$$
\mathbf{B B}^{H}=\mathbf{I}_{K},
$$

which involves further mathematical developments as described in Appendix A.

Before starting with the forward link case, let us remark that $\mathbf{B}^{\star}$ only needs statistical channel knowledge in order to be computed. Moreover, its design does not depend on $\alpha$ as long as the resulting rank of $\breve{\mathbf{Z}}$ is equal to $K$. Indeed, the value of $\alpha$ affects only on the resulting SMSE $\mathrm{RL}_{\mathrm{R}}$. This is due to the optimization of an upper bound of the problem instead of the problem itself. Now, let us proceed with the forward link optimization. 
In the forward link the optimization problem can be formulated as follows

$$
\begin{aligned}
& \min _{\mathbf{B}} \operatorname{trace}\left(\left(\gamma^{2} \mathbf{B} \breve{\mathbf{Z}} \mathbf{B}^{H}+\frac{K}{P_{\mathrm{FL}}} \mathbf{I}_{K}\right)^{-1}\right) \\
& \text { s.t. } \mathbf{B B}^{H}=\mathbf{I}_{K} .
\end{aligned}
$$

In can be observed that the optimal solution of (39) is (36). The sketch of the proof is similar to the one presented previously for the return link and; thus, we only comment it. The idea is to check whether the term $\frac{K}{P_{\mathrm{FL}}}$ does not influence the optimal value of (39) which can be easily observed in appendix A. Consequently, neither the scaling factor due to the channel variations $\gamma$ does not influence the optimization. Remarkably, this derivation is different from the one presented in our preliminary work in [13], because this paper considers the forward and return link optimizations.

Note that the beamforming scheme depends on $\overline{\mathbf{H}}$ so that the system designer needs to be aware of it in advance. This information can be obtained through current deployments or estimations. In addition, the robust beamforming design has the same eigenvectors as the nominal channel matrix $\overline{\mathbf{H}} \overline{\mathbf{H}}^{H}$. In other words, the presented robust design only considers eigenvalue variations due to the different user positions. In the next section, the impact on the eigenvectors is analyzed.

\section{First Order Perturbation Analysis}

Considering the derivations of the previous section, obtaining a robust on-board beamforming matrix leads to computing an accurate upper-bound of $\mathbf{Z}$ considering the different sources of perturbation. This section completes the upper-bound by including an additional impact of the perturbation errors.

A complete perturbation model can be described as

$$
\begin{aligned}
\mathbf{Z}= & \left(\overline{\mathbf{U}}_{s}+\Delta \mathbf{U}_{s}\right)\left(\overline{\boldsymbol{\Sigma}}_{s}+\Delta \boldsymbol{\Sigma}_{s}\right)\left(\overline{\mathbf{U}}_{s}+\Delta \mathbf{U}_{s}\right)^{H} \\
& +\left(\overline{\mathbf{U}}_{n}+\Delta \mathbf{U}_{n}\right)\left(\overline{\boldsymbol{\Sigma}}_{n}+\Delta \boldsymbol{\Sigma}_{n}\right)\left(\overline{\mathbf{U}}_{n}+\Delta \mathbf{U}_{n}\right)^{H},
\end{aligned}
$$

where the $\mathbf{U}$ denotes the matrix containing the eigenvectors and $\boldsymbol{\Sigma}$ is a diagonal matrix which contains the eigenvalues. Sub-index $s$ denotes the non-zero signal space whereas $n$ the signal space that is spanned by the zero valued eigenvalues (i.e. the null space of $\mathbf{Z}$ ). All $\Delta \mathbf{U}_{s}, \Delta \boldsymbol{\Sigma}_{s}, \Delta \mathbf{U}_{n}, \Delta \boldsymbol{\Sigma}_{n}$ are generated by a perturbed version of $\overline{\mathbf{Z}}$ :

$$
\mathbf{Z}=\overline{\mathbf{Z}}+\Delta \mathbf{Z}
$$

where

$$
\overline{\mathbf{Z}}=\overline{\mathbf{H}} \overline{\mathbf{H}}^{H},
$$

and

$$
\Delta \mathbf{Z}=\overline{\mathbf{H}} \Delta^{H}+\Delta \overline{\mathbf{H}}^{H}+\Delta \Delta^{H} .
$$

Under this context, $\overline{\mathbf{U}}$ denotes the eigenvector of the nominal matrix $\overline{\mathbf{Z}}$ whereas $\overline{\boldsymbol{\Sigma}}$ a matrix containing its eigenvalues. The other matrices with the $\Delta$. prefix denote the corresponding perturbation matrices.

The previous section has implicitly considered two assumptions. First, it has been assumed that the channel variations do not modify the dimension of the null space so that $\Delta \boldsymbol{\Sigma}_{n}$ remains as a zero matrix. Second, it has been assumed that $\Delta \mathbf{U}_{s}=0$, which might not be true in certain cases [21]. The aim of this section is to consider the effect of this later perturbation in order to obtain a more realistic version of $\mathbf{Z}$ than the presented in the previous section, $\widehat{\mathbf{Z}}$.

This novel approximation of $\mathbf{Z}, \widehat{\mathbf{Z}}$, considers both perturbations at both eigenvalues and eigenvectors $\left(\Delta \mathbf{U}_{s}\right)$. Mathematically,

$$
\widehat{\mathbf{Z}}=\left(\overline{\mathbf{U}}_{s}-\mathbf{P}\right)\left(\overline{\mathbf{\Sigma}}-\epsilon_{H} \mathbf{I}\right)\left(\overline{\mathbf{U}}_{s}-\mathbf{P}\right)^{H},
$$

where $\mathbf{P}$ is a semidefinite positive matrix that has the same dimensions of $\mathbf{U}_{s}$. Note that it is essential to obtain a matrix $\mathbf{P}$ that collapses the maximum of the eigenvectors perturbation. In the following we propose a solution in order to properly design the on-board beamforming when eigenvector perturbations are present.

Proposal The beamforming matrix that takes into account both the eigenvalues and eigenvector perturbation can be written as

$$
\widehat{\mathbf{B}^{*}}=\widehat{\mathbf{U}}=\overline{\mathbf{U}}_{s}-\left(\epsilon_{H} \overline{\mathbf{U}}_{s} \widehat{\mathbf{R}}+\epsilon_{H} \overline{\mathbf{U}}_{n} \overline{\mathbf{U}}_{n}^{H} \overline{\mathbf{U}}_{s} \overline{\boldsymbol{\Sigma}}_{s}^{-1}\right),
$$

where

$$
\widehat{\mathbf{R}}=\mathbf{D} \circ\left(\mathbf{U}_{s}^{H} \mathbf{U}_{s} \overline{\boldsymbol{\Sigma}}+\overline{\boldsymbol{\Sigma}} \mathbf{U}_{s}^{H} \mathbf{U}_{s}\right),
$$

and the $g, f$-th entry of $\mathbf{D}$ is

$$
\frac{1}{\lambda_{f}-\lambda_{g}}
$$

for $f \neq g$ and $\lambda_{f}$ for $f=1, \ldots, N$ denote the eigenvalues of $\overline{\mathbf{H}} \overline{\mathbf{H}}^{H}$.

Proof: See Appendix B.

Note that for this case, the eigenvectors of the beamforming matrix take a different value from the nominal matrix. In addition, the larger $\alpha$ the more different are the eigenvectors from the nominal channel matrix ones. However, $\alpha$ cannot take any arbitrary value. Indeed, the permissible perturbation value is dictated by the fact that the resulting matrix containing the eigenvectors shall be semidefinite positive. As a result, $\epsilon_{H}$ shall hold

$$
\overline{\mathbf{U}}_{s} \geq\left(\epsilon_{H} \overline{\mathbf{U}}_{s} \widehat{\mathbf{R}}+\epsilon_{H} \overline{\mathbf{U}}_{n} \overline{\mathbf{U}}_{n}^{H} \overline{\mathbf{U}}_{s} \overline{\boldsymbol{\Sigma}}_{s}^{-1}\right) .
$$

It is important to remark that $\widehat{\mathbf{B}^{*}}$ is not a unitary matrix and; thus, it is not an efficient solution of the optimization problem (39) as it does not satisfies the constraints. However, we take the heuristic approach of electing $\widehat{\mathbf{B}^{*}}$ processing, even though the solution is not unitary since it is obtained from a more detailed description of the perturbation errors impact. This solution is validated in the simulation section and it is observed that it presents a slightly better performance than the B $^{*}$ solution.

As we have already seen, the beam generation process both on the forward and return links leads to the same matrix $\mathbf{B}$, which is fixed. Now, it is time to compare the benefits of this design in front of the current beamforming deployments. 


\section{Simulation Results}

In order to show the performance of our proposal, this section presents a numerical evaluation of the conceived technique. Our baseline scenario is an array fed reflector antenna and matrix $\mathbf{B}$ that have been provided by ESA in the framework of a study on next generation multibeam satellite systems. ${ }^{2}$ The number of feeds is assumed to be $N=155$ and $K=100$ beams that are covering the whole Europe area.

Results have been averaged over a total of 1000 user link channel realizations. Note that, only atmospheric fading due to rain effect is considered in the user link channel and further refinements of the channel are neglected. This simple characterization is useful for the intended comparisons and it is a general practice in the evaluation of multibeam satellite systems.

The randomness of the channel is due to the user positions which are assumed to be uniformly distributed within the beams. In addition, we will assume that each user employs all available spectrum and the atmospheric fading is modelled as in [22].

Recall that, full frequency reuse among beams and noiseless feeder link have been considered in this work. In the sequel, we compute different performance metrics. First, the SINR for each user after employing interference mitigation techniques among users is presented. Then, with that SINR value, the throughput is inferred according to DVB-RCS and DVB-S2 standards for the return and forward links, respectively [23], [24]. Furthermore, the simulation results also provide the SINR statistics. In this case, the instantaneous availability indicator for the $k$-th user is given by

$$
A_{k}=g\left(\operatorname{SINR}_{k}\right)
$$

which is equal to 0 if the user link is unavailable (i.e, if the instantaneous SINR is lower than that required by the lowest modcod for the return link, i.e. $\operatorname{SINR}_{k}<1.7 \mathrm{~dB}$, and for the forward link, i.e. $\operatorname{SINR}_{k}<-2.72 \mathrm{~dB}$ ) and is equal to 1 otherwise. We also present the Shannon capacity ${ }^{3}$ obtained from the user SINR,

$$
C_{\text {Shannon }}=\log _{2}(1+\text { SINR }),
$$

and assuming that interference is treated as Gaussian noise. This measurement serves us to see the potential of our work independently of the satellite standard modulations and channel coding both for the forward and return links.

Another performance metric to be considered is the fairness among beams. Note that this is of great interest for satellite operators where near to equal achievable data rates per beam are the target. For this purpose, we present the throughput index of dispersion, defined as

$$
\text { Index of Dispersion }=\frac{\sigma_{\mathrm{Th}}}{\mu_{\mathrm{Th}}},
$$

where $\sigma_{\mathrm{Th}}$ and $\mu_{\mathrm{Th}}$ correspond to the variance and the mean of the user throughputs, respectively. This metric provides an

\footnotetext{
${ }^{2}$ http://satnex4.org/

${ }^{3}$ Of course, we refer to the use of the Shannon formula instead of the channel capacity.
}

indicator of how the data rates are dispersed with respect to the mean. The larger the index of dispersion is, the lower the fairness the system achieves.

For a best practice, as upper bound for the achievable rates we consider only on-ground processing at the gateway (i.e. no on-board processing) as it is described in [11]. From the return link point of view, the received signal (4), which is based on this on-ground scenario, is rewritten as

$$
\mathbf{y}_{\mathrm{RL}}=\mathbf{T}_{\text {on-ground }}^{H}(\mathbf{H s}+\mathbf{n}),
$$

where

$$
\mathbf{T}_{\text {on-ground }}=\mathbf{H}\left(\mathbf{H H}^{H}+\mathbf{I}_{K}\right)^{-1}
$$

denotes the LMMSE detector filter at the gateway. Note that the linear processing is similar to (12) but in this case it has been assumed that no beamforming is done. Considering the forward link, the received signal by the user terminals with this on-ground technique can be represented as

$$
\mathbf{y}_{\mathrm{FL}}=\mathbf{H}^{T} \mathbf{T}_{\text {on-ground }} \mathbf{x}+\mathbf{w} .
$$

It is important to remark that although large data rates can be obtained if all the processing is carried out on ground, the required feeder link spectral resources severely increase, leading to a possibly inefficient system.

To sum up, in order to test the validity of the derived theoretical results in section IV, we compute the spectral efficiency of the following multibeam satellite system using precoding and detection algorithms for forward and return links respectively:

- B based on a geographical reasoning (reference). ${ }^{4}$

- $\mathbf{B}^{*}$ proposed by this study in (36).

- $\widehat{\mathbf{B}}^{*}$ proposed by this study in (45).

- On ground processing (upper bound).

In the sequel, the results are separated into two different subsections, return and forward link. In this context, the same fixed optimal design of on-board beamforming matrix is computed since this optimal design depends on the right eigen vector of channel average matrix, $\overline{\mathbf{H}}$. This is computed empirically considering the aforementioned 1000 channel user realizations.

\section{A. Return Link}

The return link operates at $30 \mathrm{GHz}$, and is based on DVBRCS standard [23] and we target a Packet Error Rate (PER) of $10^{-7}$. Figure 3 depicts the evolution of the total average throughput (bits/symbol) as a function of the user EIRP $(\beta)$ for different scenarios. Although by means of using the DVB-RCS standard the obtained throughput gain is limited when the Shannon capacity is considered, higher gains are obtained with respect to the reference scenario. In other words, other modcods design would improve the benefits of the proposed technique with respect to the reference scenario. Note that the proposed robust design that consider the eigenvector perturbation improves the system throughput with

\footnotetext{
${ }^{4}$ This beam generation process has attended geographical reasons, so that ESA confirmed all Europe achieves a sufficient signal power strength.
} 


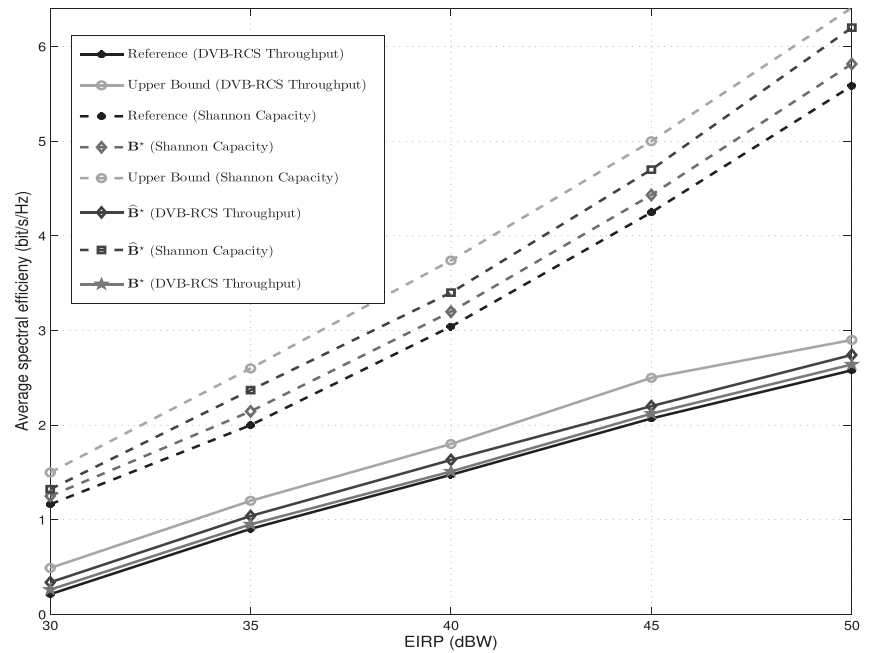

Fig. 3. Return link throughput values over different user $\operatorname{EIRP}(\beta)$.

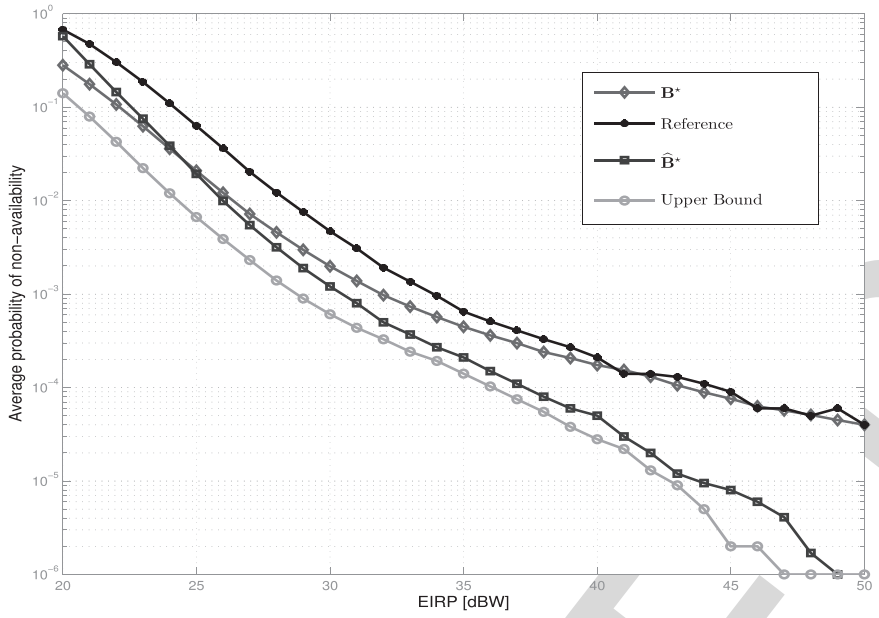

Fig. 4. Return link availability.

respect to the design that only considers eigenvalue variations. Indeed, our proposal is approaching the upper bound of the on ground design.

The corresponding availability probability is also provided in Figure 4. In this case, our proposal also improves the reference scenario, leading to an increase of the system availability. Remarkably, the fairness among beams is also improved as it is depicted in Figure 5. Lower values of dispersion index are obtained with our technique with respect to the reference design.

Finally, we study the impact of the channel variations on the beamforming design. Bearing in mind that $\alpha$ in (33) determines this variation, we compute this value and we present its corresponding average throughput values in Figure 6. The values of $\alpha$ are selected so that the feasibility of $\mathrm{MSE}_{\mathrm{RL}}$ in (32) holds. It implies that

$$
\left(\overline{\bar{\Sigma}}-\epsilon_{H} \mathbf{I}_{N}\right)_{i i} \geq 0 \quad \forall i=1, \ldots, N .
$$

For a large value of $\alpha$ the matrix (55) might become semidefinite negative and; thus, changes the nature of the problem. In order to avoid this, $\alpha$ has to be checked so that the matrix (55) always remains semidefinite positive. It is observed

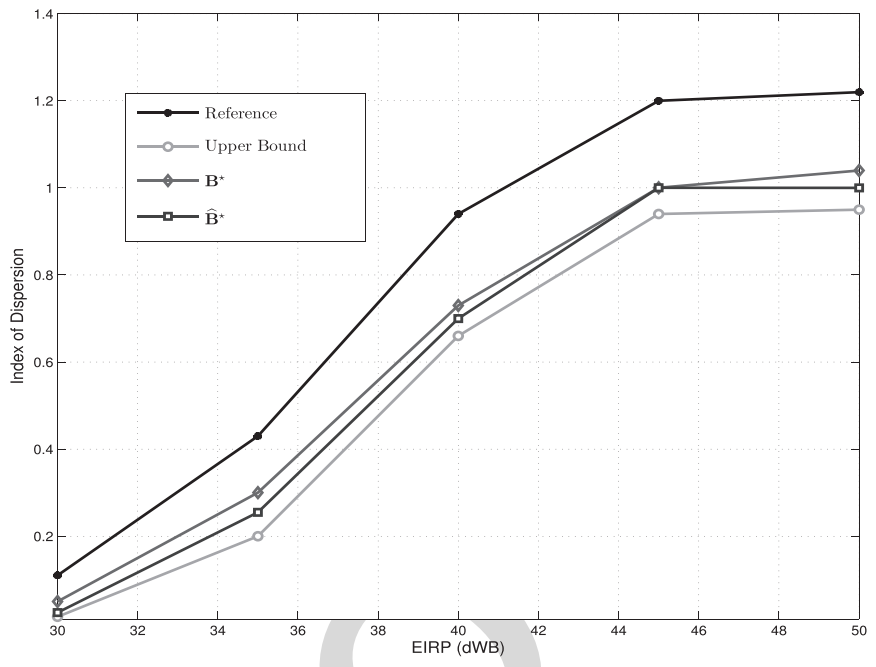

Fig. 5. Return link throughput index of dispersion.

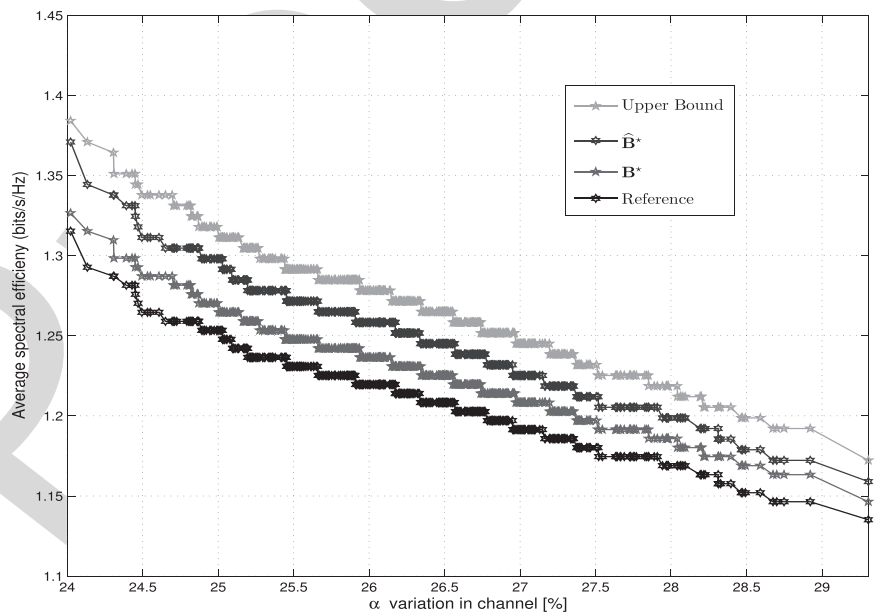

Fig. 6. Return link throughput with respect to channel variations. Note that $\alpha$ determines maximum variation of the channel at each time instant, i.e. $\|\mathbf{\Delta}\| \leq \alpha$.

that the larger $\alpha$ values, the less the throughput is obtained due to the channel mismatch.

\section{B. Forward Link}

The forward link is assumed to operate at $20 \mathrm{GHz}$ and is based on DVB-S2 standard with a PER of $10^{-6}$. Note that the working points were extrapolated from the PER curves reported in the DVB-S2 guidelines document [24]. Based on [24], it is possible to find a relationship between the required received SINR and the spectral efficiency achieved by DVB-S2 standard.

The results are presented for the total bandwidth and as a function of the total available power denoted by $P_{F L}$. Figure 7 depicts the achieved results of spectral efficiency and Figure 8 shows the availability of the users in the forward link. Clearly, the proposed techniques perform better than the benchmark system and again the robust design based on the eigenvector perturbations behaves better than the one that only considers the eigenvalues.

The expected result of throughputs in Figure 7 is justified by the availability in Figure 8. In other words, the system with 


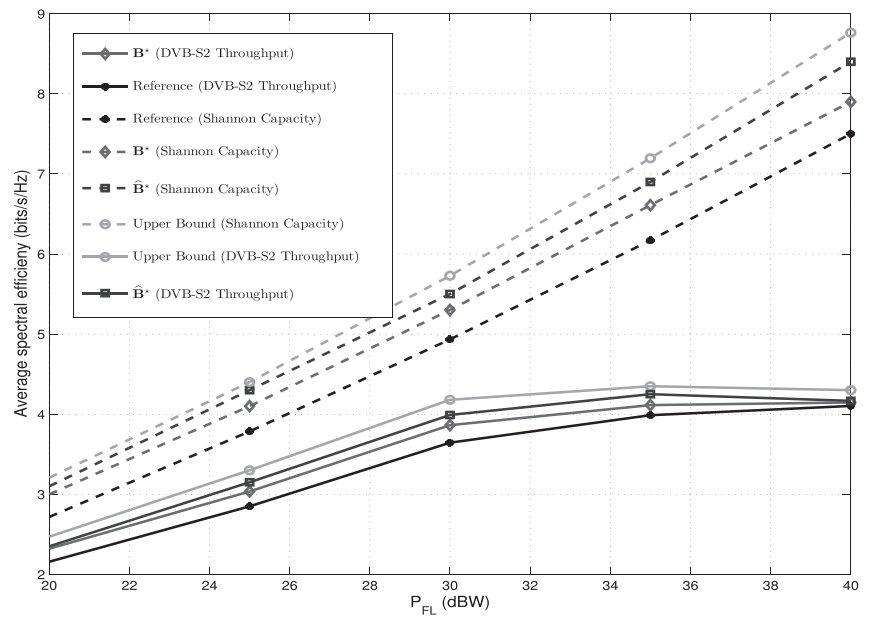

Fig. 7. Forward link throughput values.

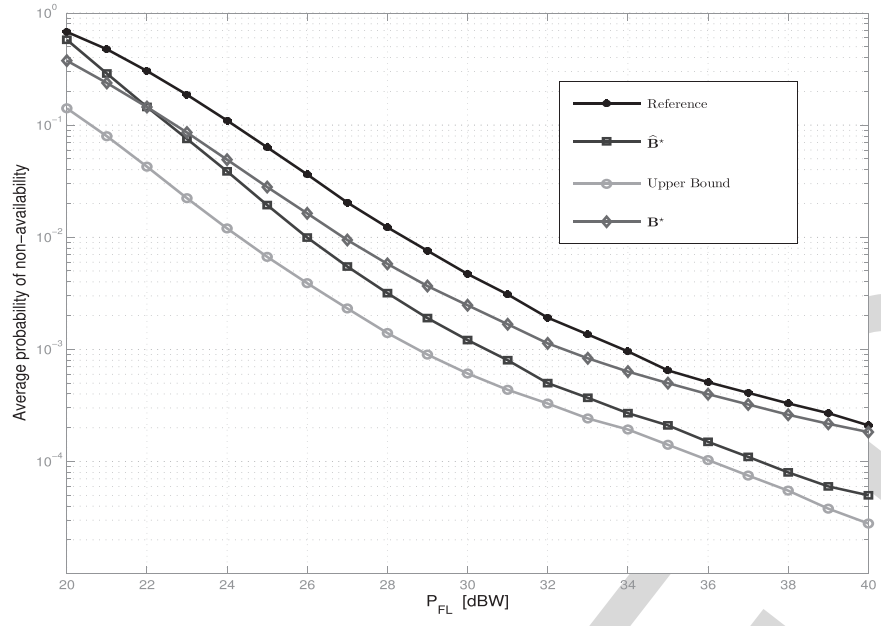

Fig. 8. Forward link availability.

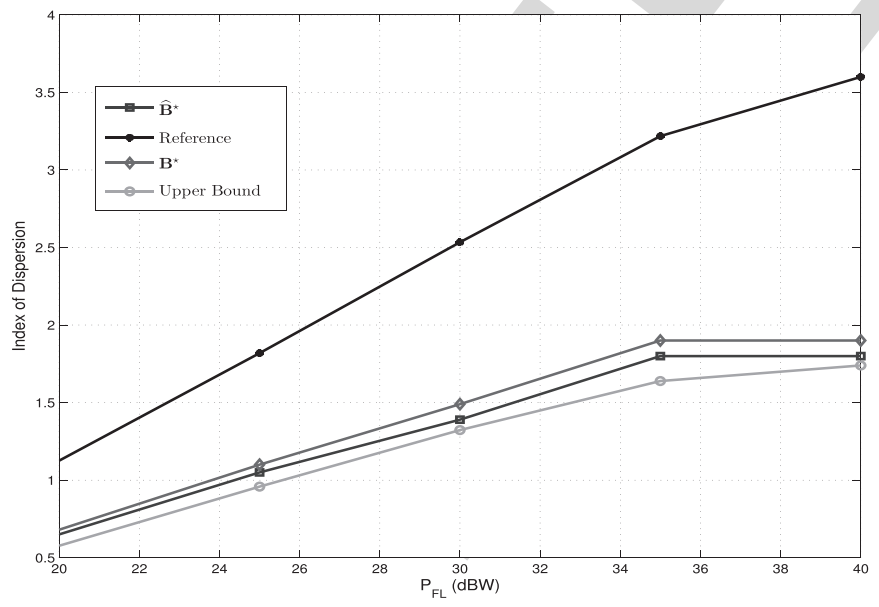

Fig. 9. Forward link throughput index of dispersion. new proposed design of $\widehat{\mathbf{B}}^{*}$ is closer to upper bound scenario than the reference. Moreover, the impact of channel variations can be observed in Figure 10. It is clear that our proposal results in higher throughputs even when the channel variations are high. Remarkably, for the forward link the performance difference is higher than the one obtained in the return link. Note that, similar to the return link, the values of $\alpha$ are selected

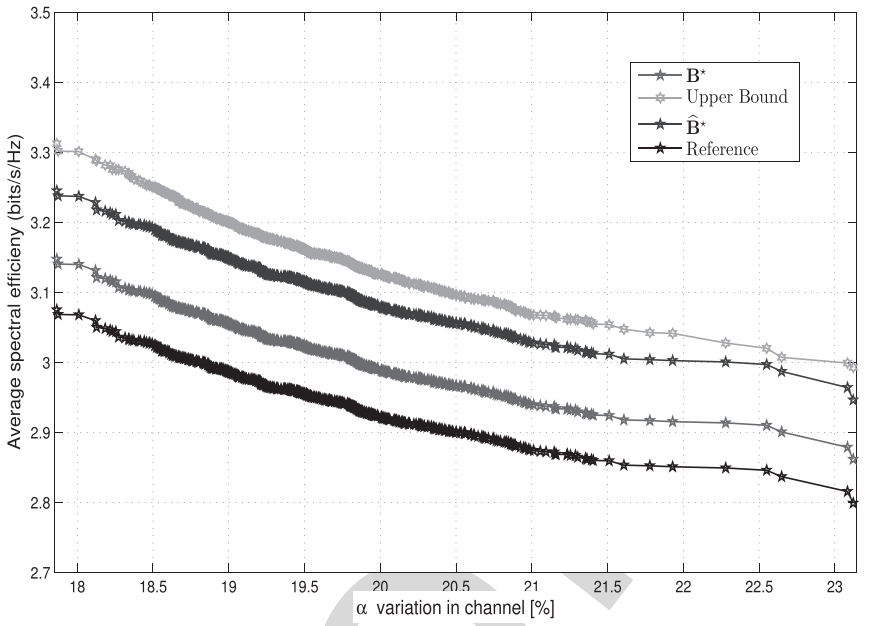

Fig. 10. Forward link throughput with respect to channel variations. Note that $\alpha$ determines maximum variation of the channel at each time instant, i.e. $\|\mathbf{\Delta}\| \leq \alpha$
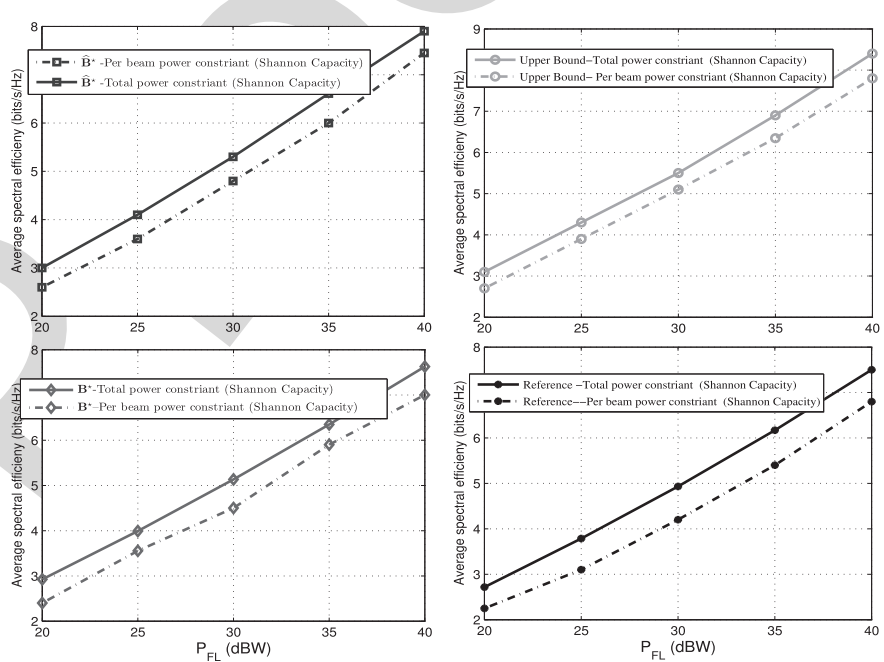

Fig. 11. Forward link throughput with per antenna power constraints.

so that the feasibility of $\mathrm{MSE}_{\mathrm{FL}}$ in (32) holds. Figure 11 describes the effect of per antenna power constraints. It can be observed that the through is severely reduced considering this power allocation. In addition, it has been considered that the available power is equally distributed among feeds. The dispersion index among users is analysed and represented in Figure 9. For this case, the dispersion values are even higher for the reference scenario and our approach leads to higher fairness between beams.

Finally, in order to compare the different satellite architectures we describe in Table I the total capacity values and required feeder link bandwidth for few satellite architectures. The values are obtained for a transmit power of $30 \mathrm{dBW}$ for the forward link with MMSE precoding. Moreover, the hybrid architecture uses the proposed on-board processing based on the eigenvector perturbation scheme.

The parameter $\eta$ is defined as the ratio between the total satellite capacity (i.e. the sum of the rates of all beams) and the feeder link bandwidth, and the colour is defined as the frequency reuse factor within the beam coverage area. In light of the above table, it is evident that on-ground precoding with 
TABLE I

Comparison of Total CAPACITY Versus FeEder LinK BANDWIDTH REQUIREMENTS FOR DIFFERENT MULTIBEAM SATELLITE ARCHITECTURES

\begin{tabular}{|c|c|c|c|}
\hline Architecture & $\begin{array}{c}\text { Total Capacity } \\
(\mathrm{Gbit} / \mathrm{s})\end{array}$ & $\begin{array}{c}B_{\text {feeder link }} \\
(\mathrm{GHz})\end{array}$ & $\eta$ \\
$(\mathrm{bit} / \mathrm{s} / \mathrm{Hz})$
\end{tabular}

2 colours is the most efficient architecture in terms of $\eta$. However, this architecture offers lower system capacity (107 Gbit/s) with respect to the hybrid and on-ground with full frequency reuse (158 and $137 \mathrm{Gbit} / \mathrm{s}$ respectively). As a result, the hybrid architecture is the most adequate solution considering the future traffic demand forecasts. Although the numerical details are not included in this work, the same conclusion can be obtained for the return link part.

It is important to remark that even through the computation of $\eta$ depends on the beampattern, $N$ and $K$ as a general statement we can infer that, given a satellite user available bandwidth (500 MHz for this case), our proposed on-board beamforming technique offers a large throughput yet maintaining a low feeder link bandwidth requirement compared to the other architectures with only on-ground processing. Remarkably, although the 2-colour plus precoding solution offers a larger $\eta$, its system capacity yields to a lower value compared to the hybrid case and; thus, this later solution is the most adequate for next generation multibeam satellite systems.

Finally, the on-board beamforming entails an additional payload processing compared for the pure on-ground approach. This complexity increase could limit its applicability in future systems. Therefore, the system designer could eventually opt to a pure on-ground architecture whose payload complexity is lower compared to the hybrid case. In this context, attending to the system capacity increase, the best option is the one colour plus precoding approach. On the other hand, the 2 colour plus precoding case is the one that offers the largest feeder link efficiency.

\section{CONCLUSION}

This paper proposes a design of non-channel adaptive beam generation process that increases the system throughput compared to the conventional existing techniques in both forward and return link of a multibeam satellite system. The design is based on an upper bound approximation of the worst case SMSE, which results to be the same for both forward and return links, leading to a large reduction of the payload complexity. The robust approximation relies on a first perturbation model which results tighter than current robust designs. Moreover, the simulation results also have shown the potential advantage of the considered design in order to increase the total system throughput. As a consequence, this new approach could become a breakthrough in the design of the next satellite systems, which so far have designed the onboard beamforming only based on geographical information.

\section{APPENDIX A}

The goal is to prove, the proposed optimal design of $\mathbf{B}$ in (36) can minimize the upper-bound of $\mathrm{SMSE}_{\mathrm{RL}}$ in (35). First, by employing the eigenvalue decomposition of $\breve{\mathbf{Z}}$ in (32), problem (35) can be rewritten as

$$
\begin{aligned}
& \min _{\mathbf{M}_{R L}} \operatorname{trace}\left(\left(\mathbf{I}_{K}+\mathbf{M}_{R L} \mathbf{D}_{R L} \mathbf{M}_{R L}^{H}\right)\right)^{-1} \\
& \text { s.t. } \mathbf{M}_{R L} \mathbf{M}_{R L}^{H}=\mathbf{I}_{K},
\end{aligned}
$$

with the following definitions

$$
\mathbf{M}_{R L} \triangleq \mathbf{B} \overline{\mathbf{U}}
$$

and,

$\mathbf{D}_{R L} \triangleq\left(\overline{\boldsymbol{\Sigma}}-\epsilon_{H} \mathbf{I}_{N}\right)^{+}=\left(\begin{array}{cc}\left(\overline{\boldsymbol{\Sigma}}_{1: K}-\epsilon_{H} \mathbf{I}_{K}\right)^{+} & \mathbf{0}_{K \times(N-K)} \\ \mathbf{0}_{(N-K) \times K} & \mathbf{0}_{(N-K) \times(N-K)}\end{array}\right)$,

where $\overline{\boldsymbol{\Sigma}}$ has only $K$ non-zero eigenvalues, as $\overline{\mathbf{H}} \overline{\mathbf{H}}^{H}$ has rank equal to $K$. Actually, the problem (56) can be written as

$$
\begin{aligned}
& \min _{\mathbf{M}_{R L}} \sum_{i=1}^{K} \frac{1}{1+\lambda_{i}\left(\mathbf{M}_{R L} \mathbf{D}_{R L} \mathbf{M}_{R L}^{H}\right)} \\
& \text { s.t. } \mathbf{M}_{R L} \mathbf{M}_{R L}^{H}=\mathbf{I}_{K},
\end{aligned}
$$

where $\lambda_{i}($.$) denotes the i$-th largest eigenvalue of the respective matrix. Obviously, $\mathbf{M D M}{ }^{H}$ is a hermitian matrix whose eigenvalues are always positive. Then, it follows that

$$
g\left(\lambda_{i}\right)=\frac{1}{1+\lambda_{i}\left(\mathbf{M}_{R L} \mathbf{D}_{R L} \mathbf{M}_{R L}^{H}\right)} \quad i=1, \ldots, K ;
$$

is convex function on $\lambda_{i}\left(\mathbf{M}_{R L} \mathbf{D}_{R L} \mathbf{M}_{R L}^{H}\right)$. By using [25, Th. 3.C.1], we have that

$$
\begin{aligned}
\phi(\lambda) & =\sum_{i=1}^{K} \frac{1}{1+\lambda_{i}\left(\mathbf{M}_{R L} \mathbf{D}_{R L} \mathbf{M}_{R L}^{H}\right)} \\
& =\sum_{i=1}^{K} g\left(\lambda_{i}\left(\mathbf{M}_{R L} \mathbf{D}_{R L} \mathbf{M}_{R L}^{H}\right)\right),
\end{aligned}
$$

where $\lambda=\left(\lambda_{1}\left(\mathbf{M}_{R L} \mathbf{D}_{R L} \mathbf{M}_{R L}^{H}\right), \ldots, \lambda_{K}\left(\mathbf{M}_{R L} \mathbf{D}_{R L} \mathbf{M}_{R L}^{H}\right)\right)^{T}$, and $\phi($.$) is a schur-convex function operator. On other hand,$ [25, Th. B.1] proved that

$$
\mathbf{d} \prec \lambda,
$$

where $\mathbf{d}($.$) represents K \times 1$ vector formed by the diagonal elements of the matrix $\mathbf{M}_{R L} \mathbf{D}_{R L} \mathbf{M}_{R L}^{H}$, i.e. $\mathbf{d}=$ $\left(d_{1}\left(\mathbf{M}_{R L} \mathbf{D}_{R L} \mathbf{M}_{R L}^{H}\right), \ldots, d_{K}\left(\mathbf{M}_{R L} \mathbf{D}_{R L} \mathbf{M}_{R L}^{H}\right)\right)^{T}$. Finally, combining of (62) with the schur convexity of $\phi($.$) , we have that$ $\phi(\mathbf{d}) \leq \phi(\lambda)$, i.e.

$$
\sum_{i=1}^{K} \frac{1}{1+d_{i}\left(\mathbf{M}_{R L} \mathbf{D}_{R L} \mathbf{M}_{R L}^{H}\right)} \geq \sum_{i=1}^{K} \frac{1}{1+\lambda_{i}\left(\mathbf{M}_{R L} \mathbf{D}_{R L} \mathbf{M}_{R L}^{H}\right)}
$$


Moreover, the equality in (63) is reached whenever $\mathbf{M}_{R L} \mathbf{D}_{R L} \mathbf{M}_{R L}^{H}$ is diagonal. To this end, it is clear that $\mathbf{M}$ has to be diagonal such that

$$
\mathbf{M}_{R L}=\left[\begin{array}{ll}
\mathbf{I}_{K} & \mathbf{0}_{K \times(N-K)}
\end{array}\right] .
$$

Note that this differs to the developments in $[20, \mathrm{Sec}$. V] where the diagonal elements are optimized for different objective functions. Our approach is to meet the constraint in (56).

Given (57), it implies that $\mathbf{B}$ has to be made of the $K$ first rows of the matrix $\overline{\mathbf{U}}^{H}$, that is

$$
\mathbf{B}=\overline{\mathbf{U}}_{1: K}^{H},
$$

and concludes the proof.

\section{APPENDIX B}

The starting point of the derivation is the upper bound of $\mathbf{Z}$ obtained when considering only the eigenvalues variation

$$
\overline{\mathbf{U}}_{s}\left(\overline{\boldsymbol{\Sigma}}-\epsilon_{H} \mathbf{I}\right) \overline{\mathbf{U}}_{s}^{H},
$$

where for this case we additionally consider the perturbation on the eigenvectors as

$$
\left(\overline{\mathbf{U}}_{s}+\Delta \mathbf{U}_{s}\right)\left(\overline{\boldsymbol{\Sigma}}-\epsilon_{H} \mathbf{I}\right)\left(\overline{\mathbf{U}}_{s}+\Delta \mathbf{U}_{s}\right)^{H} .
$$

Note that the derivation in order to obtain Theorem 1, remains the same even though the perturbation over the eigenvectors is considered. In [21] it is presented that the perturbation on the eigenvectors take the form of

$$
\Delta \mathbf{U}_{s}=\overline{\mathbf{U}}_{s} \mathbf{R}+\overline{\mathbf{U}}_{n} \overline{\mathbf{U}}_{n}^{H} \Delta \mathbf{Z} \overline{\mathbf{U}}_{s} \overline{\boldsymbol{\Sigma}}_{s}^{-1}
$$

where

$$
\mathbf{R}=\mathbf{D} \circ\left(\mathbf{U}_{s}^{H} \Delta \mathbf{Z} \mathbf{U}_{s} \overline{\boldsymbol{\Sigma}}+\bar{\Sigma} \mathbf{U}_{s}^{H} \Delta \mathbf{Z}^{H} \mathbf{U}_{s}\right),
$$

and the $g, f$-th entry of $\mathbf{D}$ is

$$
\frac{1}{\lambda_{f}-\lambda_{g}},
$$

for $f \neq g$ and $\lambda_{f}$ for $f=1, \ldots, N$ denote the eigenvalues of $\overline{\mathbf{H}} \overline{\mathbf{H}}^{H}$.

The aim of this derivation is to substitute the unknown matrix $\Delta \mathbf{Z}$ by the known matrix $\epsilon_{H} \mathbf{I}$ which models its maximum perturbation value. This derivation entails certain assumptions of the definite positiveness of different matrices that might not hold in general. In any case, the aim of the following work is to motivate and relate the proposed solution with the eigenvector matrix perturbation.

First, we want to show that

$$
\Delta \mathbf{U}_{s} \leq \overline{\mathbf{U}}_{s} \mathbf{R}+\epsilon_{H} \overline{\mathbf{U}}_{n} \overline{\mathbf{U}}_{n}^{H} \overline{\mathbf{U}}_{s} \overline{\boldsymbol{\Sigma}}_{s}^{-1},
$$

in case the following inequality holds

$$
\Delta \mathbf{Z} \leq \epsilon_{H} \mathbf{I} .
$$

In order to show this, we shall assume

$$
\overline{\mathbf{U}}_{n} \overline{\mathbf{U}}_{n}^{H} \Delta \mathbf{Z} \overline{\mathbf{U}}_{s} \overline{\boldsymbol{\Sigma}}_{s}^{-1} \leq \epsilon_{H} \overline{\mathbf{U}}_{n} \overline{\mathbf{U}}_{n}^{H} \overline{\mathbf{U}}_{s} \overline{\boldsymbol{\Sigma}}_{s}^{-1} .
$$

Considering (73) it can be obtained (71).
Additionally, we have to assume

$\mathbf{U}_{s}^{H} \Delta \mathbf{Z} \mathbf{U}_{s} \overline{\boldsymbol{\Sigma}}+\bar{\Sigma} \mathbf{U}_{s}^{H} \Delta \mathbf{Z}^{H} \mathbf{U}_{s} \leq \epsilon_{H} \mathbf{U}_{s}^{H} \mathbf{U}_{s} \overline{\boldsymbol{\Sigma}}+\epsilon_{H} \overline{\boldsymbol{\Sigma}} \mathbf{U}_{s}^{H} \mathbf{U}_{s}$.

The following lemma is required for obtaining the result.

Lemma 2: For any complex matrix $\mathbf{K}$ and two square complex matrices $\mathbf{A} \geq \mathbf{B} \geq \mathbf{0}$, it holds that

$$
\mathbf{K} \circ \mathbf{A} \geq \mathbf{K} \circ \mathbf{B} \text {. }
$$

Proof: This can be derived from in [26, Th. 17].

With this last result it is possible to write the following

$$
\Delta \mathbf{U}_{s} \leq \overline{\mathbf{U}}_{s} \widehat{\mathbf{R}}+\epsilon_{H} \overline{\mathbf{U}}_{n} \overline{\mathbf{U}}_{n}{ }^{H} \overline{\mathbf{U}}_{s} \overline{\boldsymbol{\Sigma}}_{s}^{-1},
$$

where

$$
\widehat{\mathbf{R}}=\mathbf{D} \circ\left(\mathbf{U}_{s}^{H} \Delta \mathbf{Z} \mathbf{U}_{s} \overline{\boldsymbol{\Sigma}}+\bar{\Sigma} \mathbf{U}_{s}^{H} \Delta \mathbf{Z}^{H} \mathbf{U}_{s}\right) .
$$

Note that we have assumed

$$
\epsilon_{H} \mathbf{U}_{s}^{H} \mathbf{U}_{s} \overline{\boldsymbol{\Sigma}}+\epsilon_{H} \overline{\boldsymbol{\Sigma}} \mathbf{U}_{s}^{H} \mathbf{U}_{s} \geq 0 .
$$

In this context, Lemma 2 can be applied.

Considering the inequality in (74) jointly with (76), we can write

$$
\Delta \mathbf{U}_{s} \leq \overline{\mathbf{U}}_{s} \hat{\mathbf{R}}+\epsilon_{H} \overline{\mathbf{U}}_{n} \overline{\mathbf{U}}_{n}{ }^{H} \overline{\mathbf{U}}_{s} \overline{\boldsymbol{\Sigma}}_{s}^{-1},
$$

where

$$
\widehat{\mathbf{R}}=\mathbf{D} \circ\left(\epsilon_{H} \mathbf{U}_{s}^{H} \mathbf{U}_{s} \overline{\boldsymbol{\Sigma}}+\epsilon_{H} \overline{\boldsymbol{\Sigma}} \mathbf{U}_{s}^{H} \mathbf{U}_{s}\right) .
$$

The right side of the inequality in (79) is the one presented in the proposal.

\section{ACKNOWLEDGMENT}

The authors would like to thank the anonymous reviewers whose comments extremely increased the quality of the paper.

\section{REFERENCES}

[1] J.-D. Gayrard, "Terabit Satellite: Myth or reality?" in Proc. 1st Int. Conf. Adv. Satellite Space Commun., (SPACOMM), Jul. 2009, pp. 1-6.

[2] G. Zheng, S. Chatzinotas, and B. E. Ottersten, "Generic optimization of linear precoding in multibeam Satellite systems," IEEE Trans. Wireles Commun., vol. 11, no. 6, pp. 2308-2320, Jun. 2012.

[3] A. Gharanjik, B. Rao, P.-D. Arapoglou, and B. Ottersten, "Gateway switching in Q/V band Satellite feeder links," IEEE Commun. Lett., vol. 17 , no. 7 , pp. 1384-1387, Jul. 2013.

[4] V. Joroughi, M. A. Vazquez, and A. Perez-Neira, "Multiple gateway precoding with per feed power constraints for multibeam Satellite systems," in Proc. 20th Eur. Wireless Conf., May 2014, pp. 1-7.

[5] G. Zheng, S. Chatzinotas, and B. Ottersten, "Multi-gateway cooperation in multibeam Satellite systems," in Proc. IEEE 23rd Int. Symp. Pers Indoor Mobile Radio Commun. (PIMRC), Sep. 2012, pp. 1360-1364.

[6] M. Schneider, C. Hartwanger, and H. Wolf, "Antennas for multiple spot beam Satellites," CEAS Space J., vol. 2, nos. 1-4, pp. 59-66, 2011. [Online]. Available: http://dx.doi.org/10.1007/s12567-011-0012-z

[7] J. Tronc, P. Angeletti, N. Song, M. Haardt, J. Arendt, and G. Gallinaro, "Overview and comparison of on-ground and on-board beamforming techniques in mobile Satellite service applications," Int. J. Satellite Commun. Netw., no. 4, pp. 291-308, 2013. [Online]. Available: http://dx.doi.org/10.1002/sat.1049

[8] P. Angeletti, N. Alagha, and S. D'Addio, "Space/ground beamforming techniques for Satellite communications," in Proc. IEEE Antennas Propag. Soc. Int. Symp., Jul. 2010, pp. 1-4.

[9] L. Cottatellucci et al., "Interference mitigation techniques for broadband Satellite system," in Proc. ICSSC, San Diego, CA, USA, Jun. 2006, p. 5348. [Online]. Available: http://www.eurecom.fr/publication/1886 
[10] V. Boussemart, M. Berioli, F. Rossetto, and M. Joham, "On the achievable rates for the return-link of multi-beam Satellite systems using successive interference cancellation," in Proc. MILITARY Commun. Conf. MILCOM, Nov. 2011, pp. 217-223.

[11] J. Arnau, B. Devillers, C. Mosquera, and A. Perez-Neira, "Performance study of multiuser interference mitigation schemes for hybrid broadband multibeam Satellite architectures," EURASIP J. Wireless Commun. Netw., vol. 2012, no. 1, p. 132, 2012. [Online]. Available: http://jwcn.eurasipjournals.com/content/2012/1/132

[12] B. Devillers, A. Perez-Neira, and C. Mosquera, "Joint linear precoding and beamforming for the forward link of multi-beam broadband Satellite systems," in Proc. IEEE Global Telecommun. Conf. (GLOBECOM), Dec. 2011, pp. 1-6.

[13] V. Joroughi, B. Devillers, M. Á. Vázquez, and A. I. Pérez-Neira, "Design of an on-board beam generation process for the forw ard link of a multi-beam broadband Satellite system," in Proc. IEEE Global Commun. Conf. (Globecom), Dec. 2013, pp. 2921-2926.

[14] M. Aloisio and P. Angeletti, "Multi-amplifiers architectures for power reconfigurability," in Proc. IEEE Int. Vac. Electron. Conf. (IVEC), May 2007, pp. 1-2.

[15] C. B. Peel, B. M. Hochwald, and A. L. Swindlehurst, "A vectorperturbation technique for near-capacity multiantenna multiuser communication-Part I: Channel inversion and regularization," IEEE Trans. Commun., vol. 53, no. 1, pp. 195-202, Jan. 2005.

[16] J. Arnau-Yanez et al., "Hybrid space-ground processing for highcapacity multi-beam Satellite systems," in Proc. IEEE Global Telecommun. Conf. (GLOBECOM), Dec. 2011, pp. 1-6.

[17] J. Wang and D. P. Palomar, "Worst-case robust MIMO transmission with imperfect channel knowledge," IEEE Trans. Signal Process., vol. 57, no. 8, pp. 3086-3100, Aug. 2009.

[18] D. Palomar, "A unified framework for communications through MIMO channels," Ph.D. dissertation, Tech. Univ. Catalonia, Barcelona, Spain, 2003.

[19] A. Pascual-Iserte, D. P. Palomar, A. I. Perez-Neira, and M. A. Lagunas, "A robust maximin approach for MIMO communications with imperfect channel state information based on convex optimization," IEEE Trans. Signal Process., vol. 54, no. 1, pp. 346-360, Jan. 2006.

[20] D. P. Palomar, J. M. Cioffi, and M. A. Lagunas, "Joint Tx-Rx beamforming design for multicarrier MIMO channels: A unified framework for convex optimization," IEEE Trans. Signal Process., vol. 51, no. 9, pp. 2381-2401, Sep. 2003.

[21] J. Liu, X. Liu, and X. Ma, "First-order perturbation analysis of singular vectors in singular value decomposition," IEEE Trans. Signal Process. vol. 56, no. 7, pp. 3044-3049, Jul. 2008.

[22] M. S. Alouini, S. A. Borgsmiller, and P. G. Steffes, "Channel characterization and modeling for Ka-band very small aperture terminals," Proc. IEEE, vol. 85, no. 6, pp. 981-997, Jun. 1997.

[23] H. Skinnemoen, C. Rigal, A. Yun-Garcia, L. Erup, N. Alagha, and A. Ginesi, "Dvb-rcs2 overview," Int. J. Satellite Commun. Netw., vol. 31, no. 5, pp. 201-217, 2013.

[24] A. Morello and V. Mignone, "DVB-S2: The second generation standard for Satellite broad-band services," Proc. IEEE, vol. 94, no. 1, pp. 210-227, Jan. 2006.

[25] A. W. Marshall and I. Olkin, Inequalities: Theory of Majorization and Its Application. San Diego, CA, USA: Academic, 1979.

[26] C. R. Johnson, "Partitioned and Hadamard product matrix inequalities," J. Res. Nat. Bureau Standards, vol. 83, no. 6, pp. 3044-3049, Nov. 1978.

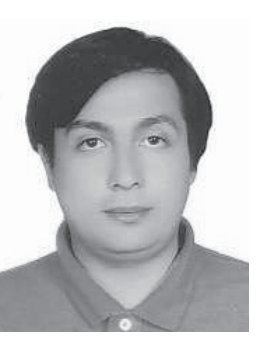

Vahid Joroughi was born in Mianeh, Iran, in 1980 $\mathrm{He}$ received the bachelor's and master's degrees in electrical engineering in 2003 and 2009, respectively. He joined Iran Telecommunication Company (ITC) as a Switching Manager in order to participate in the NEAX project jointly supported by ITC and NEC Company.

From 2012 to 2015, he received the FPU-UPC Grant to conduct the Ph.D. degree jointly with the Center Tecnólogic de Telecomunicacions de Catalunya and Universidad Politécnica de Catalunya, Barcelona. His Ph.D. program was done in the context of different projects provided by European Space Agency on the topic of multibeam satellite communication network. He currently holds a post-doctoral position with the Universidade de Vigo.

His research interest includes satellite communications with special emphasis on MIMO satellite infrastructure, statistical/array signal processing, and cooperative communication.

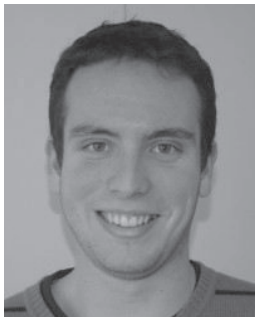

Miguel Ángel Vázquez was born in Palma de Mallorca, Spain, in 1986. He received the Telecommunication Engineering degree from the TelecomBCN Technical University of Catalonia (UPC), the master's degree (Hons.) with a focus on wireless communication in 2012, and the Ph.D. degree in telecommunications from UPC in 2014. His Ph.D. thesis was on beamforming and power control in spectrum sharing systems. He joined the Centre Tecnològic de les Telecomunicacions de Catalunya in 2010. He has been granted a Marie Curie Fellowship in the context of a FP7 European Project (SWAP). He has participated in different EU-funded projects including the FP7, the ARTEMISA, the CELTIC, and the H2020, on the topics of Internet of Things and smart grid, and other industrial contracts with local companies and the European Space Agency. His research interests include statistical and array signal processing, spectrum sharing wireless communications, licensed shared access networks, and satellite communications. He is co-organizing the IEEE ASMSSPSC 2016 Conference and the IEEE S3P Summer School in signal processing for satellite communications.

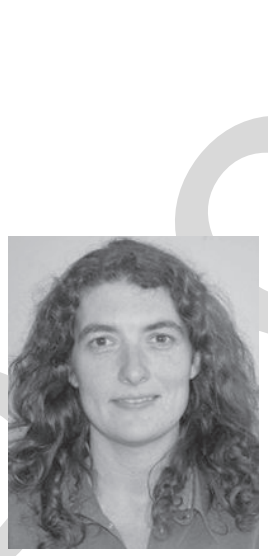

Ana I. Pérez-Neira (S'92-M'95-SM'01) created the UPC Doctoral School in 2011. She was the ViceDirector of Research with UPC from 2010 to 2013 She is currently a Full Professor with the Signa Theory and Communication Department, Technical University of Catalonia (UPC). She is also a Scientific Coordinator with the Centre Tecnològic de les Telecomunicacions de Catalunya. She is also the Coordinator of the European Project SANSA and the Network of Excellence on satellite communications, financed by the European Space Agency: SatnexIV. She has been the leader of 20 projects and has participated in over 50 (ten for European Space Agency). She has authored 50 journal papers (20 related to Satcom) and over 200 conference papers (20 invited). She has co-authored four books and five patents (one on Satcom). She has been a guest editor on five special issues. Her research topic is signal processing for communications and currently she is working in multi-antenna and multicarrier signal processing for satellite communications and wireless systems. She was on the Board of Directors of ETSETB (Telecom Barcelona) from 2000 to 2003. Since 2008, she has been a member of the European Signal Processing Association BoD. Since 2010, she has been a member of the IEEE Signal Processing Theory and Methods. She has been the General Chairman of the IWCLD09, the EUSIPCO11, the EW14, and the IWSCS14. She has participated in the organization of the ESA conference 1996 and the SAM04. She will be the General Chair of ASMS16 and the Chair of next IEEE ICASSP20. She is currently an Editor of the IEEE TRANSACTIONS ON SIgNAL PROCESSING and the EURASIP Signal Processing and Advances in Signal Processing.

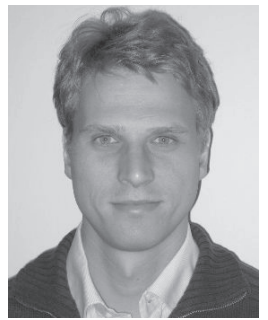

Bertrand Devillers received the degree in electrical engineering and the Ph.D. degree from the Université Catholique de Louvain (UCL), Belgium, in 2004 and 2009, respectively. From 2004 to 2008, he was a Research Fellow of the F.R.S.-FNRS with the Communications and Remote Sensing Laboratory, Digital Communication Group, UCL, Belgium. From 2009 to 2013, he was a Research Associate with the Centre Tecnològic de les Telecomunicacions de Catalunya, Barcelona, Spain. From 2008 to 2009, he was a Research Assistant with UCL, Belgium. His research interests are in communication theory and signal processing for digital terrestrial and satellite communications, multiple input multiple output systems, multicarrier, and cyclically prefixed single carrier modulations, and energy efficiency. 


\section{AUTHOR QUERIES}

\section{AUTHOR PLEASE ANSWER ALL QUERIES}

PLEASE NOTE: We cannot accept new source files as corrections for your paper. If possible, please annotate the PDF proof we have sent you with your corrections and upload it via the Author Gateway. Alternatively, you may send us your corrections in list format. You may also upload revised graphics via the Author Gateway.

AQ:1 = Please be advised that per instructions from the Communications Society this proof was formatted in Times Roman font and therefore some of the fonts will appear different from the fonts in your originally submitted manuscript. For instance, the math calligraphy font may appear different due to usage of the usepackage[mathcal]euscript. We are no longer permitted to use Computer Modern fonts.

AQ:2 = Please provide the postal codes for "Universidade de Vigo, Centre Tecnològic de les

Telecomunicacions de Catalunya, and Universitat Politècnica de Catalunya."

AQ:3 = Please provide the current affiliation of "Bertrand Devillers."

AQ:4 = Note that if you require corrections/changes to tables or figures, you must supply the revised files, as these items are not edited for you.

AQ:5 = Please confirm the volume no. for ref. [11].

AQ:6 = Please provide the department name for ref. [18].

AQ:7 = Please confirm whether the edits made in the sentence "He joined... satellite communication network." are OK. 